\title{
Generic formalism for homogenization of coupled behavior: Application to magnetoelectroelastic behavior
}

\author{
Romain Corcolle, ${ }^{*}$ Laurent Daniel, ${ }^{\dagger}$ and Frédéric Bouillault ${ }^{\ddagger}$ \\ Laboratoire de Génie Electrique de Paris, CNRS UMR8507; SUPELEC; UPMC Univ Paris 06; Univ Paris-Sud; \\ 11 rue Joliot-Curie, Plateau de Moulon, F-91192 Gif-sur-Yvette Cedex, France
}

\begin{abstract}
Multiphysics couplings are at the origin of many applications. In order to design devices with optimal coupling effects, advanced modeling tools are necessary. In this paper a generic formalism for the homogenization of coupled magnetoelectrothermoelastic behavior is proposed. It is based on the decomposition in several contributions of the magnetic, electric, thermal, and mechanical fields. This decomposition makes it possible to use the classical homogenization tools developed in the framework of uncoupled behavior. The method is illustrated on the calculation of the effective properties of a magnetoelectric composite made of piezomagnetic and piezoelectric phases.
\end{abstract}

\section{INTRODUCTION}

Coupled behavior is a long-standing domain of interest in physics. The possibility of applications such as sensors or actuators, relying on these multiphysics coupling effects have made these materials receive more and more attention in the past years. In order to design properly these different applications, advanced modeling tools are needed. Phenomenological macroscopic approaches can give a first description. ${ }^{1}$ These approaches proved to be very efficient in the case of homogeneous materials. But a promising issue is the development of sensors and actuators made of composite materials. The properties of each constituent can then be combined to obtain optimal macroscopic properties. In those cases, all the more if the composition of the composite material has to be optimized, a phenomenological macroscopic approach is not suitable anymore. This is particularly true when the observed macroscopic coupling effect is not observed in any of the constituent of the composite (see the example of magnetoelectric composite in Sec. IV).

If the heterogeneity of the material is to be accounted for in the definition of the macroscopic constitutive law, a classical approach is the use of finite element modeling. This choice has been applied, for example, in the case of electrostrictive, ${ }^{2}$ magnetostrictive, ${ }^{3}$ or magnetoelectric ${ }^{4,5}$ composites. Another convenient method is the use of homogenization techniques, leading to much lower computational times. Some authors applied these techniques in the case of electrostrictive, ${ }^{6-8}$ magnetostrictive, ${ }^{9,10}$ or magnetoelectric ${ }^{11,12}$ behavior. These approaches are often based upon the adaptation to coupled phenomena of the classical homogenization tools designed in the framework of uncoupled behavior.

We show in this paper that an appropriate decomposition (Fig. 1) of the state variables of a coupled problem allows us to keep unchanged the classical-uncoupled-homogenization rules. The constitutive laws used in the problem are then very close to the classical-uncoupled ones, and the relative contribution of the coupled phenomena to the macroscopic response can be clearly identified.

In Sec. II, the constitutive laws are written, using the proposed decomposition of the state variables. In Sec. III, we develop in that framework the localization-homogenization rules, unchanged compared to the classical-uncoupled ones. In Sec. IV, an application of the proposed homogenization model is done on a piezomagnetic and piezoelectric composite. That composite exhibits a macroscopic magnetoelectric effect, whereas none of the phases exhibit such an effect. This behavior is shown to be well captured by the model and compared to another heterogeneous models.

\section{CONSTITUTIVE LAWS}

Coupled effects are often observed in the macroscopic behavior of materials. These effects are the manifestation of the interaction between mechanics, magnetism, electricity, thermics, etc. For example, the piezomagnetic behavior is observed when the apparent permeability of a material depends on the level of stress or when the strain depends on the level of the magnetic induction. The constitutive law of the material links the applied loading to the response of the material. The loading can be a mechanical strain, a magnetic,

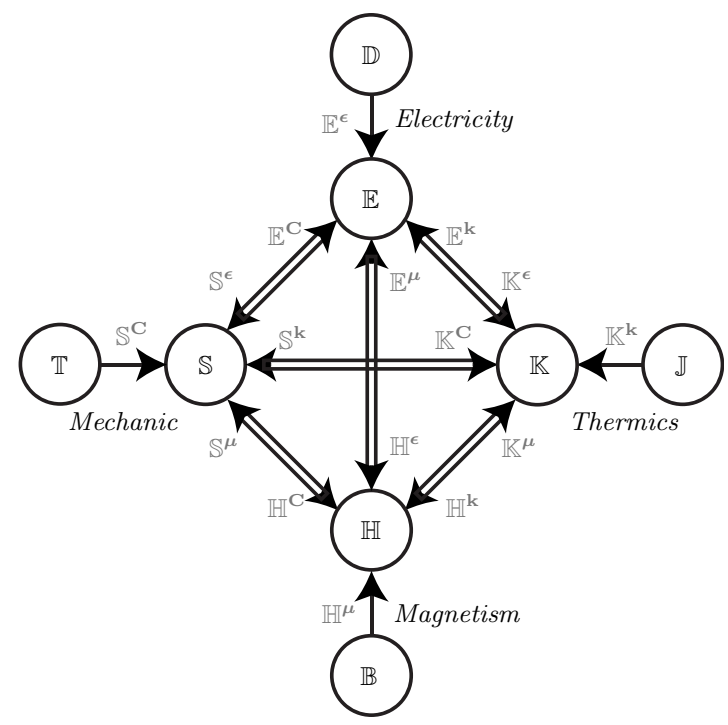

FIG. 1. Coupling effects decomposition. 
electric or thermal field, or any combination of them. The response is then a stress, a magnetic, electric or thermal induction, or any combination of them. The constitutive laws are usually written separately for each uncoupled behavior. It is convenient to write a global constitutive law, intrinsically including the coupling effects. It is the objective of this section. We first summarize the classical expression for uncoupled behavior. We then define the general constitutive law from these expressions.

\section{A. Uncoupled constitutive laws}

\section{Mechanics}

For uncoupled-mechanical behavior, the dual state variables are the strain tensor $\mathrm{S}$ (linked to the displacement field $\mathbf{u}, \mathrm{S}=1 / 2\left(\nabla \mathbf{u}+{ }^{t} \nabla \mathbf{u}\right)$, the operator ${ }^{t} \mathbf{X}$ denoting a transposition operation over $\mathbf{X}$ ), and the stress tensor $\mathbb{T}$. In a purely elastic problem, the relationship between these tensors (Hooke's law) is written as

$$
\mathbb{T}=\mathbf{C} \cdot \mathrm{S},
$$

where $\mathrm{S}$ and $\mathbb{T}$ are second-order tensors $(3 \times 3)$ and $\mathbf{C}$ is the fourth-order stiffness tensor $(3 \times 3 \times 3 \times 3)$. The modified Voigt notation (Voigt-Mandel notation) can be used, thanks to the symmetries exhibited by these tensors (see Appendix A). Then, $S$ and $\mathbb{T}$ are represented by $(6 \times 1)$ vectors and $\mathbf{C}$ is represented by a $(6 \times 6)$ matrix.

\section{Magnetism}

For uncoupled-magnetic behavior, the dual state variables are the magnetic field $\mathbb{H}$ (linked to the magnetic potential $\phi$ in absence of currents, $H=-\nabla \phi$ ) and the magnetic induction $B$. In a purely magnetic problem, the relationship between these fields is written as

$$
\mathrm{B}=\boldsymbol{\mu} \cdot \mathrm{H},
$$

where $\mathbb{H}$ and $\mathbb{B}$ are vectors $(3 \times 1)$ and $\boldsymbol{\mu}$ is the second-order permeability tensor $(3 \times 3)$.

\section{Electricity}

For uncoupled-electric behavior, the dual state variables are the electric field $\mathbb{E}$ (linked to the electric potential $\varphi$, $\mathbb{E}=-\nabla \varphi)$ and the electric induction $\mathbb{D}$. In a purely electric problem, the relationship between these fields is written as

$$
\mathrm{D}=\boldsymbol{\epsilon} \cdot \mathbb{E},
$$

where $\mathbb{E}$ and $D$ are vectors $(3 \times 1)$ and $\boldsymbol{\epsilon}$ is the second-order permittivity tensor $(3 \times 3)$.

\section{Thermics}

For uncoupled-thermal behavior, the dual state variables are the thermal field $\mathbb{K}$ (linked to the temperature $\theta, \mathbb{K}$ $=-\nabla \theta$ ) and the thermal induction $\mathbb{J}$. In a purely thermal problem, the relationship between these fields (Fourier's law) is written as

$$
\mathrm{J}=\mathbf{k} \cdot \mathbb{K},
$$

where $\mathbb{K}$ and $J$ are vectors $(3 \times 1)$ and $\mathbf{k}$ is the second-order thermal-conductivity tensor $(3 \times 3)$.

\section{Global constitutive law}

For a material with no coupling effects, the global constitutive law is just the superposition of the previous laws,

$$
\left(\begin{array}{c}
\mathrm{T} \\
\mathrm{B} \\
\mathrm{D} \\
\mathrm{J}
\end{array}\right)=\left(\begin{array}{cccc}
\mathbf{C} & \mathbf{0} & \mathbf{0} & \mathbf{0} \\
\mathbf{0} & \boldsymbol{\mu} & \mathbf{0} & \mathbf{0} \\
\mathbf{0} & \mathbf{0} & \boldsymbol{\epsilon} & \mathbf{0} \\
\mathbf{0} & \mathbf{0} & \mathbf{0} & \mathbf{k}
\end{array}\right) \cdot\left(\begin{array}{c}
\mathrm{S} \\
\mathbb{H} \\
\mathbb{E} \\
\mathbb{K}
\end{array}\right) .
$$

The response vector ${ }^{t}(\mathbb{T} B \quad D J)$ is constituted of fields with null divergence $[\nabla \cdot \mathrm{T}=\mathbf{0}$ (in the absence of volumic forces), $\nabla \cdot \mathrm{B}=0, \nabla \cdot \mathrm{D}=0$ (in the absence of electric charges), and $\nabla \cdot \mathbb{J}=0$ (in the absence of own heating sources)]. On the other hand, the loading vector ${ }^{t}(\mathrm{~S} H \mathbb{E} \mathbb{K})$ is constituted of fields that each derives from a potential.

\section{B. Coupled constitutive laws}

\section{Global constitutive law}

For a material exhibiting coupling phenomena, the global constitutive law includes coupling parameters $\boldsymbol{\alpha}$,

$$
\left(\begin{array}{c}
\mathbb{T} \\
\mathbb{B} \\
\mathbb{D} \\
J
\end{array}\right)=\left(\begin{array}{cccc}
\mathbf{C} & \boldsymbol{\alpha}^{\mathbf{C} \mu} & \boldsymbol{\alpha}^{\mathbf{C \epsilon}} & \boldsymbol{\alpha}^{\mathbf{C k}} \\
\boldsymbol{\alpha}^{\mu \mathbf{C}} & \boldsymbol{\mu} & \boldsymbol{\alpha}^{\mu \epsilon} & \boldsymbol{\alpha}^{\mu \mathbf{k}} \\
\boldsymbol{\alpha}^{\epsilon \mathbf{C}} & \boldsymbol{\alpha}^{\epsilon \mu} & \boldsymbol{\epsilon} & \boldsymbol{\alpha}^{\epsilon \mathbf{k}} \\
\boldsymbol{\alpha}^{\mathbf{k C}} & \boldsymbol{\alpha}^{\mathbf{k} \boldsymbol{\mu}} & \boldsymbol{\alpha}^{\mathbf{k} \epsilon} & \mathbf{k}
\end{array}\right) \cdot\left(\begin{array}{c}
\mathrm{S} \\
\mathbb{H} \\
\mathbb{E} \\
\mathbb{K}
\end{array}\right)
$$

This generic form for the constitutive laws is compact, but it is not well suited to the use of classical homogenization tools. Indeed homogenization models generally provide some so-called localization operators (for example, based on Eshelby inclusion problem in mechanics) linking the local fields to the macroscopic loading. But these localization operators usually apply to uncoupled behavior. In the case of coupling effects, some authors proposed to define some new coupled Eshelby tensors accounting for magnetoelectromechanical coupling. ${ }^{13}$ This tensor depends on the nature of the coupling and on the distribution of the phases, meaning that it has to be computed for every studied configuration. We propose to keep using the well-known classical homogenization models: the adaptation to coupled behavior relying on an appropriate decomposition of the fields. This method is inspired from the usual strain decomposition in mechanics. ${ }^{14,15}$

\section{Mechanics}

In the case of coupled behavior, we divide the strain into several contributions. For a magnetoelectrothermomechanical coupling, the total strain tensor $\mathrm{S}$ can be decomposed in the following way:

$$
\mathrm{S}=\mathrm{S}^{\mathbf{C}}+\mathrm{S}^{\boldsymbol{\mu}}+\mathrm{S}^{\boldsymbol{\epsilon}}+\mathrm{S}^{\mathbf{k}}
$$

where $\mathrm{S}^{\mathbf{C}}, \mathrm{S}^{\boldsymbol{\mu}}, \mathrm{S}^{\boldsymbol{\epsilon}}$, and $\mathrm{S}^{\mathbf{k}}$ represent, respectively, the purely mechanical, the magnetic, the electric, and the thermal strain tensors. The purely mechanical strain tensor $\mathrm{S}^{\mathbf{C}}$ is linked to the stress tensor ' $\mathbb{T}$ by the usual Hooke's law (in the case of elasticity), 


$$
\mathrm{T}=\mathbf{C} \cdot \mathrm{S}^{\mathbf{C}} .
$$

The stiffness tensor $\mathbf{C}$ (in the case of a homogeneous material) is the same that the one introduced in Eq. (6). The definition of the stiffness tensor is given by Eq. (8) and T $\neq \mathbf{C} \cdot \mathrm{S}$ in the general case.

The magnetic strain tensor $\mathrm{S}^{\mu}$ (magnetostriction) is linked to the magnetic state, the electric strain tensor $\mathbf{S}^{\boldsymbol{\epsilon}}$ (electrostriction) to the electric state, and the thermal strain tensor $S^{\mathbf{k}}$ (thermal dilatation) to the thermal state. Moreover Eq. (8) can be rewritten as

$$
\mathrm{T}=\mathbf{C} \cdot\left(\mathrm{S}-\mathrm{S}^{\boldsymbol{\mu}}-\mathrm{S}^{\boldsymbol{\epsilon}}-\mathrm{S}^{\mathbf{k}}\right) .
$$

From Eqs. (6) and (9), the different contributions to the strain can be defined as

$$
\begin{aligned}
& -\mathrm{C} \cdot \mathrm{S}^{\mu}=\alpha^{\mathrm{C} \mu} \cdot \mathbb{H}, \\
& -\mathrm{C} \cdot \mathrm{S}^{\epsilon}=\alpha^{\mathrm{C} \epsilon} \cdot \mathbb{E}, \\
& -\mathrm{C} \cdot \mathrm{S}^{\mathbf{k}}=\alpha^{\mathrm{Ck}} \cdot \mathbb{K} .
\end{aligned}
$$

\section{Magnetism}

In the case of coupled behavior, we divide the magnetic field into several contributions. For an electrothermomechanomagnetic coupling, the total magnetic field $H$ can be decomposed in the following way:

$$
H=H^{\mathbf{C}}+H^{\mu}+H^{\boldsymbol{\epsilon}}+\mathbb{H}^{\mathbf{k}},
$$

where $\mathbb{H}^{\mathbf{C}}, \mathbb{H}^{\boldsymbol{\mu}}, \mathbb{H}^{\boldsymbol{\epsilon}}$, and $\mathbb{H}^{\mathbf{k}}$ represent, respectively, the mechanical, the purely, the electric, and the thermal magnetic fields. The purely magnetic field $\mathrm{H}^{\mu}$ is linked to the magnetic induction $\mathrm{B}$ by the-uncoupled-magnetic constitutive law,

$$
\mathrm{B}=\boldsymbol{\mu} \cdot \mathbb{H}^{\mu} .
$$

The definition of the magnetic permeability is given by Eq. (12) and $\mathbb{B} \neq \boldsymbol{\mu} \cdot H$ in the general case.

The mechanical magnetic field $H^{\mathbf{C}}$ is linked to the mechanical state, the electric magnetic field $\mathbb{H}^{\boldsymbol{\epsilon}}$ to the electric state, and the thermal magnetic field $\mathbb{H}^{\mathbf{k}}$ to the thermal state. Moreover Eq. (12) can be rewritten as

$$
\mathrm{B}=\boldsymbol{\mu} \cdot\left(\mathbb{H}-\mathbb{H}^{\mathbf{C}}-\mathbb{H}^{\boldsymbol{\epsilon}}-\mathbb{H}^{\mathbf{k}}\right) .
$$

The different contributions to the magnetic field can be defined as

$$
\begin{aligned}
& -\mu \cdot \mathbb{H}^{\mathrm{C}}=\boldsymbol{\alpha}^{\mu \mathrm{C}} \cdot \mathrm{S}, \\
& -\mu \cdot \mathbb{H}^{\epsilon}=\boldsymbol{\alpha}^{\mu \epsilon} \cdot \mathbb{E}, \\
& -\mu \cdot \mathbb{H}^{\mathbf{k}}=\boldsymbol{\alpha}^{\mu \mathbf{k}} \cdot \mathbb{K} .
\end{aligned}
$$

\section{Electricity}

In the case of coupled behavior, we divide the electric field into several contributions. For a thermomechanomagnetoelectric coupling, the total electric field $\mathbb{E}$ can be decomposed in the following way:

$$
\mathbb{E}=\mathbb{E}^{\mathbf{C}}+\mathbb{E}^{\mu}+\mathbb{E}^{\epsilon}+\mathbb{E}^{\mathbf{k}},
$$

where $\mathbb{E}^{\mathbf{C}}, \mathbb{E}^{\boldsymbol{\mu}}, \mathbb{E}^{\boldsymbol{\epsilon}}$, and $\mathbb{E}^{\mathbf{k}}$ represent, respectively, the mechanical, the magnetic, the purely, and the thermal electric fields. The purely electric field $\mathbb{E}^{\boldsymbol{\epsilon}}$ is linked to the electric induction $\mathbb{D}$ by the-uncoupled-electric constitutive law,

$$
\mathbb{D}=\boldsymbol{\epsilon} \cdot \mathbb{E}^{\boldsymbol{\epsilon}},
$$

The definition of the electric permittivity is given by Eq. (16) and $\mathbb{D} \neq \boldsymbol{\epsilon} \cdot \mathbb{E}$ in the general case.

The mechanical electric field $\mathbb{E}^{\mathbf{C}}$ is linked to the mechanical state, the magnetic electric field $\mathbb{E}^{\mu}$ to the magnetic state, and the thermal electric field $\mathbb{E}^{\mathbf{k}}$ to the thermal state. Moreover Eq. (16) can be rewritten as

$$
\mathrm{D}=\boldsymbol{\epsilon} \cdot\left(\mathbb{E}-\mathbb{E}^{\mathbf{C}}-\mathbb{E}^{\mu}-\mathbb{E}^{\mathbf{k}}\right) .
$$

The different contributions to the electric field can be defined as

$$
\begin{aligned}
& -\epsilon \cdot \mathbb{E}^{\mathbf{C}}=\boldsymbol{\alpha}^{\epsilon \mathrm{C}} \cdot \mathrm{S}, \\
& -\epsilon \cdot \mathbb{E}^{\mu}=\boldsymbol{\alpha}^{\epsilon \mu} \cdot \mathbb{H}, \\
& -\boldsymbol{\epsilon} \cdot \mathbb{E}^{\mathbf{k}}=\boldsymbol{\alpha}^{\epsilon \mathbf{k}} \cdot \mathbb{K} .
\end{aligned}
$$

\section{Thermics}

In the case of coupled behavior, we divide the thermal field into several contributions. For a mechanomagnetoelectrothermal coupling, the total thermal field $\mathrm{K}$ can be decomposed in the following way:

$$
\mathbb{K}=\mathbb{K}^{\mathbf{C}}+\mathbb{K}^{\mu}+\mathbb{K}^{\boldsymbol{\epsilon}}+\mathbb{K}^{\mathbf{k}},
$$

where $\mathbb{K}^{\mathbf{C}}, \mathbb{K}^{\boldsymbol{\mu}}, \mathbb{K}^{\boldsymbol{\epsilon}}$, and $\mathbb{K}^{\mathbf{k}}$ represent, respectively, the mechanical, the magnetic, the electric, and the purely thermal fields. The purely thermal field $\mathbb{K}^{\mathbf{k}}$ is linked to the electric induction J by the Fourier's law,

$$
\mathrm{J}=\mathbf{k} \cdot \mathbb{K}^{\mathbf{k}} \text {. }
$$

The definition of the thermal conductivity is given by Eq. (20) and $J \neq \mathbf{k} \cdot \mathbb{K}$ in the general case.

The mechanical thermal field $\mathbb{K}^{\mathbf{C}}$ is linked to the mechanical state, the magnetic thermal field $\mathbb{K}^{\mu}$ to the magnetic state, and the electric thermal field $\mathbb{K}^{\boldsymbol{\epsilon}}$ to the electric state. Moreover Eq. (20) can be rewritten as

$$
J=\mathbf{k} \cdot\left(\mathbb{K}-\mathbb{K}^{\mathbf{C}}-\mathbb{K}^{\boldsymbol{\mu}}-\mathbb{K}^{\boldsymbol{\epsilon}}\right) .
$$

The different contributions to the thermal field can be defined as

$$
\begin{aligned}
& -\mathbf{k} \cdot \mathbb{K}^{\mathbf{C}}=\boldsymbol{\alpha}^{\mathbf{k C}} \cdot \mathrm{S}, \\
& -\mathbf{k} \cdot \mathbb{K}^{\mu}=\boldsymbol{\alpha}^{\mathbf{k} \mu} \cdot \mathbb{H}, \\
& -\mathbf{k} \cdot \mathbb{K}^{\epsilon}=\boldsymbol{\alpha}^{\mathbf{k} \epsilon} \cdot \mathbb{E} .
\end{aligned}
$$

\section{Summary}

The following scheme shows the coupling effects and the different contributions to the loading fields in the general 
case of magnetoelectrothermomechanical behavior. The global constitutive law can be rewritten as follows:

$$
\left(\begin{array}{c}
\mathrm{T} \\
\mathrm{B} \\
\mathrm{D} \\
\mathrm{J}
\end{array}\right)=\left(\begin{array}{cccc}
\mathbf{C} & \mathbf{0} & \mathbf{0} & \mathbf{0} \\
\mathbf{0} & \boldsymbol{\mu} & \mathbf{0} & \mathbf{0} \\
\mathbf{0} & \mathbf{0} & \boldsymbol{\epsilon} & \mathbf{0} \\
\mathbf{0} & \mathbf{0} & \mathbf{0} & \mathbf{k}
\end{array}\right) \cdot\left(\begin{array}{c}
\mathrm{S}^{\mathbf{C}} \\
\mathbb{H}^{\boldsymbol{\mu}} \\
\mathbb{E}^{\boldsymbol{\epsilon}} \\
\mathbb{K}^{\mathbf{k}}
\end{array}\right)
$$

This presentation must be completed by the coupling relations given by Eqs. (10), (14), (18), and (22). The basic decomposition given by Eqs. (7), (11), (15), and (19) is also needed. The number of relations appears to be higher than in the classical formalism, but each of them is much simpler.

\section{Example of piezoelectricity}

Piezoelectric materials exhibit a coupling between mechanics and electricity. The constitutive law is written in Eq. (24), according to the proposed formalism. This relation corresponds to Eq. (6) where the magnetoelastic, thermoelastic, thermomagnetic, thermoelectric, and magnetoelectric couplings have been canceled,

$$
\left(\begin{array}{c}
\mathrm{T} \\
\mathbb{B} \\
\mathrm{D} \\
\mathrm{J}
\end{array}\right)=\left(\begin{array}{cccc}
\mathbf{C} & \mathbf{0} & \boldsymbol{\alpha}^{\mathbf{C} \boldsymbol{\epsilon}} & \mathbf{0} \\
\mathbf{0} & \boldsymbol{\mu} & \mathbf{0} & \mathbf{0} \\
\boldsymbol{\alpha}^{\boldsymbol{\epsilon}} & \mathbf{0} & \boldsymbol{\epsilon} & \mathbf{0} \\
\mathbf{0} & \mathbf{0} & \mathbf{0} & \mathbf{k}
\end{array}\right) \cdot\left(\begin{array}{c}
\mathrm{S} \\
\mathbb{H} \\
\mathbb{E} \\
\mathbb{K}
\end{array}\right)
$$

It is interesting to compare this constitutive law to the classical piezoelectric relations given by Eqs. (25)-(28).

$$
\begin{gathered}
T_{i j}=c_{i j k l}^{E} S_{k l}-e_{k i j} E_{k}, \\
D_{i}=e_{i k l} S_{k l}+\epsilon_{i k}^{S} E_{k}, \\
S_{i j}=s_{i j k l}^{E} T_{k l}+d_{k i j} E_{k}, \\
D_{i}=d_{i k l} T_{k l}+\epsilon_{i k}^{T} E_{k}, \\
S_{i j}=s_{i j k l}^{D} T_{k l}+g_{k i j} D_{k}, \\
E_{i}=-g_{i k l} T_{k l}+\beta_{i k}^{T} D_{k}, \\
T_{i j}=c_{i j k l}^{D} S_{k l}-h_{k i j} D_{k}, \\
E_{i}=-h_{i k l} S_{k l}+\beta_{i k}^{S} D_{k} .
\end{gathered}
$$

Each of these four systems is equivalent and can be identified to Eq. (24). Considering Eq. (25), we obtain that $\mathbf{c}^{E}$ is the elastic stiffness tensor $\mathbf{C}, \boldsymbol{\epsilon}$ is the permittivity tensor $\boldsymbol{\epsilon}$, and $\mathbf{e}$ is equal to $\boldsymbol{\alpha}^{\boldsymbol{\epsilon} \mathbf{C}}$. It leads also to the additional relation that under such hypotheses, $\boldsymbol{\alpha}^{\mathbf{C} \boldsymbol{\epsilon}}=-{ }^{t} \boldsymbol{\alpha}^{\boldsymbol{\epsilon} \mathbf{C}}$. This relation is carried out from thermodynamics, dealing with reversible (linear) behavior. Equation (6) embraces a more general case and does not a priori present this kind of symmetry (or antisymmetry). It can also be noticed that an antisymmetric matrix can be changed into a symmetric one by choosing the sign of the state variables [for example, choosing $-\mathrm{S}$ instead of S in Eq. (25)]. Considering Eqs. (26)-(28), we obtain the following additional relations:

$$
\begin{gathered}
\mathbf{s}^{E}=\mathbf{C}^{-1}, \\
\boldsymbol{\epsilon}^{T}=\boldsymbol{\epsilon}+\mathbf{C}^{-1} \cdot{ }^{t} \boldsymbol{\alpha}^{\epsilon \mathbf{C}} \cdot \boldsymbol{\alpha}^{\boldsymbol{\epsilon} \mathbf{C}}, \\
\mathbf{d}=\boldsymbol{\alpha}^{\epsilon \mathbf{C}} \cdot \mathbf{C}^{-1}, \\
\mathbf{s}^{D}=\left(\mathbf{C}+{ }^{t} \boldsymbol{\alpha}^{\boldsymbol{\epsilon} \mathbf{C}} \cdot \boldsymbol{\epsilon}^{-1} \cdot \boldsymbol{\alpha}^{\boldsymbol{\epsilon} \mathbf{C}}\right)^{-1}, \\
\boldsymbol{\beta}^{T}=\left(\boldsymbol{\epsilon}+\mathbf{C}^{-1} \cdot{ }^{t} \boldsymbol{\alpha}^{\boldsymbol{\epsilon} \mathbf{C}} \cdot \boldsymbol{\alpha}^{\epsilon \mathbf{C}}\right)^{-1}, \\
\mathbf{g}=\boldsymbol{\epsilon}^{-1} \cdot \boldsymbol{\alpha}^{\boldsymbol{\epsilon} \mathbf{C}} \cdot\left(\mathbf{C}+{ }^{t} \boldsymbol{\alpha}^{\boldsymbol{\epsilon} \mathbf{C}} \cdot \boldsymbol{\epsilon}^{-1} \cdot \boldsymbol{\alpha}^{\epsilon \mathbf{C}}\right)^{-1}, \\
\mathbf{c}^{D}=\mathbf{C}+{ }^{t} \boldsymbol{\alpha}^{\boldsymbol{\epsilon} \mathbf{C}} \cdot \boldsymbol{\epsilon}^{-1} \cdot \boldsymbol{\alpha}^{\boldsymbol{\epsilon} \mathbf{C}}, \\
\boldsymbol{\beta}^{S}=\boldsymbol{\epsilon}^{-1}, \\
\mathbf{h}=\boldsymbol{\epsilon}^{-1} \cdot \boldsymbol{\alpha}^{\epsilon \mathbf{C}} .
\end{gathered}
$$

It can be noticed that the semantic ambiguousness risen on the definition of the material parameters in the classical formalism does not appear in the proposed formalism. For example, the elastic stiffness tensor definition is unique (link between stress $T$ and purely elastic strain $\mathrm{S}^{\mathbf{C}}$ ), and there is no need to distinguish a stiffness tensor measured at a constant electric induction $\mathbf{c}^{D}$ and at a constant electric field $\mathbf{c}^{E}$. Moreover, these parameters become complicated to define when nonlinearity is encountered (e.g., the so-called $\Delta E$ effect in magnetoelasticity).

Using the proposed field decomposition for $\mathrm{S}$ and $\mathbb{E}$, we get

$$
\begin{aligned}
& \mathrm{S}=\mathrm{S}^{\mathbf{C}}+\mathrm{S}^{\boldsymbol{\epsilon}}, \\
& \mathbb{E}=\mathbb{E}^{\boldsymbol{\epsilon}}+\mathbb{E}^{\mathbf{C}} .
\end{aligned}
$$

The constitutive law can be rewritten as

$$
\left(\begin{array}{l}
\mathbb{T} \\
\mathbb{D}
\end{array}\right)=\left(\begin{array}{ll}
\mathbf{C} & \mathbf{0} \\
\mathbf{0} & \boldsymbol{\epsilon}
\end{array}\right) \cdot\left(\begin{array}{l}
\mathrm{S}^{\mathbf{C}} \\
\mathbb{E}^{\boldsymbol{\epsilon}}
\end{array}\right)
$$

with additional coupling relations,

$$
\begin{gathered}
-\mathbf{C} \cdot \mathrm{S}^{\boldsymbol{\epsilon}}=-{ }^{t} \mathbf{e} \cdot \mathbb{E}, \\
-\boldsymbol{\epsilon} \cdot \mathbb{E}^{\mathbf{C}}=\mathbf{e} \cdot \mathrm{S} .
\end{gathered}
$$

These three latter equations are equivalent to any of the set of Eqs. (26)-(28). The proposed formalism is thus equivalent to the classical one in the case of the coupled behavior of homogeneous materials. But this formalism is very helpful to define the effective coupled behavior of heterogeneous materials. This point is the object of Sec. III.

\section{HOMOGENIZATION}

We now consider a heterogeneous material constituted of $n$ phases. The constitutive law for each phase $i$ is 


$$
\left(\begin{array}{c}
\mathbb{T}_{i} \\
\mathbb{B}_{i} \\
\mathbb{D}_{i} \\
\mathbb{J}_{i}
\end{array}\right)=\left(\begin{array}{cccc}
\mathbf{C}_{i} & \boldsymbol{\alpha}_{i}^{\mathbf{C} \boldsymbol{\mu}} & \boldsymbol{\alpha}_{i}^{\mathbf{C} \epsilon} & \boldsymbol{\alpha}_{i}^{\mathbf{C k}} \\
\boldsymbol{\alpha}_{i}^{\boldsymbol{\mu} \mathbf{C}} & \boldsymbol{\mu}_{i} & \boldsymbol{\alpha}_{i}^{\boldsymbol{\mu} \epsilon} & \boldsymbol{\alpha}_{i}^{\boldsymbol{\mu k}} \\
\boldsymbol{\alpha}_{i}^{\boldsymbol{\epsilon} \mathbf{C}} & \boldsymbol{\alpha}_{i}^{\boldsymbol{\epsilon} \boldsymbol{\mu}} & \boldsymbol{\epsilon}_{i} & \boldsymbol{\alpha}_{i}^{\boldsymbol{\epsilon} \mathbf{k}} \\
\boldsymbol{\alpha}_{i}^{\mathbf{k} \mathbf{C}} & \boldsymbol{\alpha}_{i}^{\mathbf{k} \boldsymbol{\mu}} & \boldsymbol{\alpha}_{i}^{\mathbf{k} \boldsymbol{\epsilon}} & \mathbf{k}_{i}
\end{array}\right) \cdot\left(\begin{array}{c}
\mathrm{S}_{i} \\
\mathrm{H}_{i} \\
\mathbb{E}_{i} \\
\mathbb{K}_{i}
\end{array}\right) .
$$

The objective is to deduce the constitutive law of the equivalent homogeneous medium (EHM). This EHM exhibits, for a given loading, the same macroscopic response than the real heterogeneous medium (RHM). The effective constitutive law is written in the form as

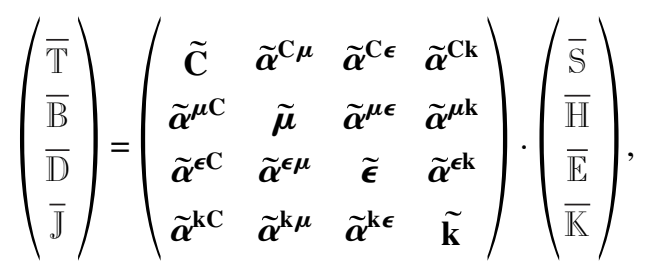

where the macroscopic loadings and responses are defined by Eq. (35),

$$
\begin{array}{ll}
\overline{\mathrm{T}}=\left\langle\mathrm{T}_{i}\right\rangle, & \overline{\mathrm{S}}=\left\langle\mathrm{S}_{i}\right\rangle, \\
\overline{\mathrm{B}}=\left\langle\mathrm{B}_{i}\right\rangle, \quad \overline{\mathrm{H}}=\left\langle\mathrm{H}_{i}\right\rangle, \\
\overline{\mathrm{D}}=\left\langle\mathrm{D}_{i}\right\rangle, \quad \overline{\mathbb{E}}=\left\langle\mathbb{E}_{i}\right\rangle, \\
\overline{\mathrm{J}}=\left\langle\mathrm{J}_{i}\right\rangle, \quad \overline{\mathrm{K}}=\left\langle\mathbb{K}_{i}\right\rangle .
\end{array}
$$

The operator $\langle\cdot\rangle$ denotes an averaging operation over the volume.

Homogenization tools have been mainly developed in the framework of uncoupled behavior. We present hereafter the homogenization model based on inclusion problems that will be used in the following.

\section{A. Inclusion-based model (uncoupled)}

For a $n$-phasic material with uncoupled behavior, the constitutive law for each phase $i$ is

$$
\left(\begin{array}{c}
\mathrm{T}_{i} \\
\mathbb{B}_{i} \\
\mathbb{D}_{i} \\
\mathrm{~J}_{i}
\end{array}\right)=\left(\begin{array}{cccc}
\mathbf{C}_{i} & \mathbf{0} & \mathbf{0} & \mathbf{0} \\
\mathbf{0} & \boldsymbol{\mu}_{i} & \mathbf{0} & \mathbf{0} \\
\mathbf{0} & \mathbf{0} & \boldsymbol{\epsilon}_{i} & \mathbf{0} \\
\mathbf{0} & \mathbf{0} & \mathbf{0} & \mathbf{k}_{i}
\end{array}\right) \cdot\left(\begin{array}{c}
\mathrm{S}_{i} \\
\mathrm{H}_{i} \\
\mathbb{E}_{i} \\
\mathbb{K}_{i}
\end{array}\right) .
$$

The effective constitutive law is

$$
\left(\begin{array}{l}
\overline{\mathrm{T}} \\
\overline{\mathrm{B}} \\
\overline{\mathrm{D}} \\
\overline{\mathrm{J}}
\end{array}\right)=\left(\begin{array}{cccc}
\overline{\mathbf{C}} & \mathbf{0} & \mathbf{0} & \mathbf{0} \\
\mathbf{0} & \overline{\boldsymbol{\mu}} & \mathbf{0} & \mathbf{0} \\
\mathbf{0} & \mathbf{0} & \overline{\boldsymbol{\epsilon}} & \mathbf{0} \\
\mathbf{0} & \mathbf{0} & \mathbf{0} & \overline{\mathbf{k}}
\end{array}\right) \cdot\left(\begin{array}{c}
\overline{\mathrm{S}} \\
\overline{\mathrm{H}} \\
\overline{\mathrm{E}} \\
\overline{\mathrm{K}}
\end{array}\right) .
$$

The effective properties are the effective elastic stiffness tensor $\overline{\mathbf{C}}$ in the purely elastic homogenization problem, the effective magnetic permeability tensor $\overline{\boldsymbol{\mu}}$ in the purely magnetic homogenization problem, the effective electric permittivity tensor $\overline{\boldsymbol{\epsilon}}$ in the purely electric homogenization problem, and the effective thermal-conductivity tensor $\overline{\mathbf{k}}$ in the purely thermal homogenization problem. These effective properties are deduced through a classical homogenization process. ${ }^{16,17}$ This process relies on the definition of localization operators, defining the local fields in phase $i$ from the knowledge of the macroscopic loading. Two types of localization operators are used. The localization tensors $\mathbf{A}$ [Eq. (38)] link the local fields deriving from potentials to the corresponding macroscopic fields, whereas the concentration tensors B [Eq. (39)] link the local fields with a null divergence to the corresponding macroscopic fields,

$$
\begin{aligned}
\mathrm{S}_{i} & =\mathbf{A}_{i}^{\mathbf{C}} \cdot \overline{\mathrm{S}}, \\
\mathrm{H}_{i} & =\mathbf{A}_{i}^{\mu} \cdot \overline{\mathrm{H}}, \\
\mathbb{E}_{i} & =\mathbf{A}_{i}^{\boldsymbol{\epsilon}} \cdot \overline{\mathrm{E}}, \\
\mathrm{K}_{i} & =\mathbf{A}_{i}^{\mathbf{k}} \cdot \overline{\mathrm{K}}, \\
\mathrm{T}_{i} & =\mathbf{B}_{i}^{\mathbf{C}} \cdot \overline{\mathrm{T}}, \\
\mathrm{B}_{i} & =\mathbf{B}_{i}^{\mu} \cdot \overline{\mathrm{B}}, \\
\mathrm{D}_{i} & =\mathbf{B}_{i}^{\boldsymbol{\epsilon}} \cdot \overline{\mathrm{D}}, \\
\mathrm{J}_{i} & =\mathbf{B}_{i}^{\mathbf{k}} \cdot \overline{\mathrm{J}} .
\end{aligned}
$$

Moreover, these localization operators verify $\left\langle\mathbf{A}_{i}\right\rangle=\left\langle\mathbf{B}_{i}\right\rangle=\mathbf{I}$ (with $\mathbf{I}$ as the identity tensor $(6 \times 6)$ in mechanics or $(3$ $\times 3)$ in magnetism, electricity, or thermics). From these localization tensors, the effective properties can be deduced,

$$
\begin{gathered}
\overline{\mathbf{C}}=\left\langle\mathbf{C}_{i} \cdot \mathbf{A}_{i}^{\mathbf{C}}\right\rangle=\left\langle\mathbf{C}_{i}^{-1} \cdot \mathbf{B}_{i}^{\mathbf{C}}\right\rangle^{-1}, \\
\overline{\boldsymbol{\mu}}=\left\langle\boldsymbol{\mu}_{i} \cdot \mathbf{A}_{i}^{\boldsymbol{\mu}}\right\rangle=\left\langle\boldsymbol{\mu}_{i}^{-1} \cdot \mathbf{B}_{i}^{\boldsymbol{\mu}}\right\rangle^{-1}, \\
\overline{\boldsymbol{\epsilon}}=\left\langle\boldsymbol{\epsilon}_{i} \cdot \mathbf{A}_{i}^{\boldsymbol{\epsilon}}\right\rangle=\left\langle\boldsymbol{\epsilon}_{i}^{-1} \cdot \mathbf{B}_{i}^{\boldsymbol{\epsilon}}\right\rangle^{-1}, \\
\overline{\mathbf{k}}=\left\langle\mathbf{k}_{i} \cdot \mathbf{A}_{i}^{\mathbf{k}}\right\rangle=\left\langle\mathbf{k}_{i}^{-1} \cdot \mathbf{B}_{i}^{\mathbf{k}}\right\rangle^{-1} .
\end{gathered}
$$

In order to build these localization operators, a homogenization model based on inclusion problems is used (see Appendix B). This method divides the problem of a $n$-phasic material into $n$-independent basic inclusion problems (Fig. 2 ). Indeed the mean response of the phase $i$ is supposed to be the same as the response of an inclusion of phase $i$ embedded in an infinite-loaded medium.

The problem of the inclusion of phase $i$ gives the localization operators $\mathbf{A}_{i}$ and $\mathbf{B}_{i}$ for phase $i$. The choice of the infinite medium properties $\left(\mathbf{C}_{\infty}, \boldsymbol{\mu}_{\infty}, \boldsymbol{\epsilon}_{\infty}\right.$, and $\left.\mathbf{k}_{\infty}\right)$ gives a degree of freedom in order to account for the influence of the microstructure of the RHM. Some particular choices enable us to retrieve some classical homogenization estimates or bounds $^{18}$ (Wiener or Voigt \& Reuss, Hashin \& Shtrikman, Mori-Tanaka, self-consistent estimate, etc.). 


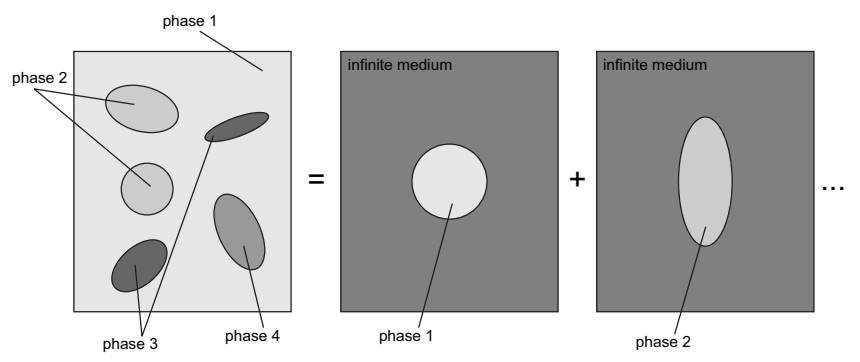

FIG. 2. Homogenization based on inclusion problems.

\section{B. Coupled problems}

If the behavior is coupled, such an approach is not directly tractable. Indeed the classical localization operators do not link the total macroscopic loading to the total local response. One method ${ }^{13}$ is to redefine the localization operators in the case of coupling effects. Our proposal is to keep the usual localization operators but combined to the use of the decomposition of the loadings presented in Sec. II B. It can be noticed that the same kind of decomposition used in Eqs. (7), (11), (15), and (19) can be used with the macroscopic fields. Moreover, a constitutive law similar to the local one [Eq. (23)] can be written macroscopically as

$$
\left(\begin{array}{c}
\overline{\mathrm{T}} \\
\overline{\mathrm{B}} \\
\overline{\mathrm{D}} \\
\overline{\mathrm{J}}
\end{array}\right)=\left(\begin{array}{cccc}
\overline{\mathbf{C}} & \mathbf{0} & \mathbf{0} & \mathbf{0} \\
\mathbf{0} & \overline{\boldsymbol{\mu}} & \mathbf{0} & \mathbf{0} \\
\mathbf{0} & \mathbf{0} & \overline{\boldsymbol{\epsilon}} & \mathbf{0} \\
\mathbf{0} & \mathbf{0} & \mathbf{0} & \overline{\mathbf{k}}
\end{array}\right) \cdot\left(\begin{array}{c}
\overline{\mathrm{S}}^{\mathbf{C}} \\
\overline{\mathrm{H}}^{\mu} \\
\overline{\mathrm{E}^{\epsilon}} \\
\overline{\mathbb{K}}^{\mathbf{k}}
\end{array}\right),
$$

where the effective properties are the ones deduced in theuncoupled problems [Eq. (40)].

But because of the coupling effects leading to local incompatibilities, one must pay attention to the fact that these decomposed macroscopic fields are not directly the mean values of the decomposed local fields [see Eqs. (42)-(45)]. Even if the total fields still respect $\overline{\mathrm{S}}=\left\langle\mathrm{S}_{i}\right\rangle, \overline{\mathrm{H}}=\left\langle\mathrm{H}_{i}\right\rangle, \overline{\mathbb{E}}=\left\langle\mathbb{E}_{i}\right\rangle$, and $\overline{\mathrm{K}}=\left\langle\mathbb{K}_{i}\right\rangle$, such a relationship does not apply to the decomposed fields. However, the macroscopic decomposed fields can be deduced from the local ones, using a modified averaging operation. This averaging operation introduces the-uncoupled-concentration tensors B (see proof in Appendix D),

$$
\begin{gathered}
\overline{\mathrm{S}}=\overline{\mathrm{S}}^{\mathbf{C}}+\overline{\mathrm{S}}^{\boldsymbol{\mu}}+\overline{\mathrm{S}}^{\boldsymbol{\epsilon}}+\overline{\mathrm{S}}^{\mathbf{k}}, \\
\overline{\mathrm{S}} \mathbf{C}=\overline{\mathbf{C}}^{-1} \cdot \overline{\mathrm{T}} \neq\left\langle\mathrm{S}_{i}^{\mathbf{C}}\right\rangle, \\
\overline{\mathrm{S}}^{\boldsymbol{\mu}}=\left\langle{ }^{t} \mathbf{B}_{i}^{\mathbf{C}} \cdot \mathrm{S}_{i}^{\boldsymbol{\mu}}\right\rangle \neq\left\langle\mathrm{S}_{i}^{\boldsymbol{\mu}}\right\rangle, \\
\overline{\mathrm{S}}^{\boldsymbol{\epsilon}}=\left\langle{ }^{\boldsymbol{t}} \mathbf{B}_{i}^{\mathbf{C}} \cdot \mathrm{S}_{i}^{\boldsymbol{\epsilon}}\right\rangle \neq\left\langle\mathrm{S}_{i}^{\boldsymbol{\epsilon}}\right\rangle, \\
\overline{\mathrm{S}}^{\mathbf{k}}=\left\langle{ }^{\left\langle\mathbf{B}_{i}^{\mathbf{C}}\right.} \cdot \mathrm{S}_{i}^{\mathbf{k}}\right\rangle \neq\left\langle\mathrm{S}_{i}^{\mathbf{k}}\right\rangle,
\end{gathered}
$$

$$
\begin{aligned}
& \overline{\mathrm{H}}=\overline{\mathbb{H}}^{\mathbf{c}}+\overline{\mathbb{H}}^{\mu}+\overline{\mathbb{H}}^{\boldsymbol{\epsilon}}+\overline{\mathrm{H}}^{\mathbf{k}}, \\
& \overline{\mathbb{H}}^{\mathbf{C}}=\left\langle{ }^{t} \mathbf{B}_{i}^{\mu} \cdot \mathbb{H}_{i}^{\mathbf{C}}\right\rangle \neq\left\langle\mathbb{H}_{i}^{\mathbf{C}}\right\rangle, \\
& \overline{\mathrm{H}}^{\boldsymbol{\mu}}=\overline{\boldsymbol{\mu}}^{-1} \cdot \overline{\mathrm{B}} \neq\left\langle\mathrm{H}_{i}^{\boldsymbol{\mu}}\right\rangle, \\
& \overline{\mathbb{H}^{\boldsymbol{\epsilon}}}=\left\langle{ }^{t} \mathbf{B}_{i}^{\mu} \cdot \mathbb{H}_{i}^{\boldsymbol{\epsilon}}\right\rangle \neq\left\langle\mathbb{H}_{i}^{\boldsymbol{\epsilon}}\right\rangle, \\
& \overline{\mathbb{H}^{k}}=\left\langle{ }^{t} \mathbf{B}_{i}^{\mu} \cdot \mathbb{H}_{i}^{\mathbf{k}}\right\rangle \neq\left\langle\mathbb{H}_{i}^{\mathbf{k}}\right\rangle, \\
& \overline{\mathbb{E}}=\overline{\mathbb{E}}^{\mathbf{C}}+\overline{\mathbb{E}}^{\mu}+\overline{\mathbb{E}}^{\epsilon}+\overline{\mathbb{E}}^{\mathbf{k}}, \\
& \overline{\mathbb{E}}^{\mathbf{C}}=\left\langle{ }^{t} \mathbf{B}_{i}^{\boldsymbol{\epsilon}} \cdot \mathbb{E}_{i}^{\mathbf{C}}\right\rangle \neq\left\langle\mathbb{E}_{i}^{\mathbf{C}}\right\rangle, \\
& \overline{\mathbb{E}}^{\mu}=\left\langle{ }^{t} \mathbf{B}_{i}^{\epsilon} \cdot \mathbb{E}_{i}^{\mu}\right\rangle \neq\left\langle\mathbb{E}_{i}^{\mu}\right\rangle, \\
& \overline{\mathbb{E}}^{\boldsymbol{\epsilon}}=\overline{\boldsymbol{\epsilon}}^{-1} \cdot \overline{\mathrm{D}} \neq\left\langle\mathbb{E}_{i}^{\boldsymbol{\epsilon}}\right\rangle, \\
& \overline{\mathbb{E}}^{\mathbf{k}}=\left\langle{ }^{t} \mathbf{B}_{i}^{\boldsymbol{\epsilon}} \cdot \mathbb{E}_{i}^{\mathbf{k}}\right\rangle \neq\left\langle\mathbb{E}_{i}^{\mathbf{k}}\right\rangle, \\
& \overline{\mathbb{K}}=\overline{\mathbb{K}}^{\mathbf{C}}+\overline{\mathbb{K}}^{\mu}+\overline{\mathbb{K}}^{\boldsymbol{\epsilon}}+\overline{\mathbb{K}}^{\mathbf{k}}, \\
& \overline{\mathbb{K}} \mathbf{C}=\left\langle{ }^{t} \mathbf{B}_{i}^{\mathbf{k}} \cdot \mathbb{K}_{i}^{\mathbf{C}}\right\rangle \neq\left\langle\mathbb{K}_{i}^{\mathbf{C}}\right\rangle, \\
& \overline{\mathbb{K}}^{\boldsymbol{\mu}}=\left\langle{ }^{t} \mathbf{B}_{i}^{\mathbf{k}} \cdot \mathbb{K}_{i}^{\boldsymbol{\mu}}\right\rangle \neq\left\langle\mathbb{K}_{i}^{\boldsymbol{\mu}}\right\rangle, \\
& \overline{\mathbb{K}}{ }^{\boldsymbol{\epsilon}}=\left\langle{ }^{t} \mathbf{B}_{i}^{\mathbf{k}} \cdot \mathbb{K}_{i}^{\boldsymbol{\epsilon}}\right\rangle \neq\left\langle\mathbb{K}_{i}^{\boldsymbol{\epsilon}}\right\rangle, \\
& \overline{\mathbb{K}}^{\mathbf{k}}=\overline{\mathbf{k}}^{-1} \cdot \overline{\mathbb{J}} \neq\left\langle\mathbb{K}_{i}^{\mathbf{k}}\right\rangle .
\end{aligned}
$$

The localization rules are similar to those defined by Hill $^{14}$ in mechanics (the tensors $\mathbf{A}$ and $\mathbf{B}$ are derived from this relation in the-uncoupled case),

$$
\begin{aligned}
& \mathbf{N}_{i}^{\mathbf{C}} \cdot \mathbf{C}_{\infty}^{-1} \cdot\left(\overline{\mathbb{T}}-\mathbb{T}_{i}\right)=\left(\mathbf{N}_{i}^{\mathbf{C}}-\mathbf{I}\right) \cdot\left(\overline{\mathrm{S}}-\mathrm{S}_{i}\right), \\
& \mathbf{N}_{i}^{\mu} \cdot \boldsymbol{\mu}_{\infty}^{-1} \cdot\left(\overline{\mathbb{B}}-\mathbb{B}_{i}\right)=\left(\mathbf{N}_{i}^{\mu}-\mathbf{I}\right) \cdot\left(\overline{\mathbb{H}}-\mathbb{H}_{i}\right), \\
& \mathbf{N}_{i}^{\epsilon} \cdot \boldsymbol{\epsilon}_{\infty}^{-1} \cdot\left(\overline{\mathbb{D}}-\mathbb{D}_{i}\right)=\left(\mathbf{N}_{i}^{\epsilon}-\mathbf{I}\right) \cdot\left(\overline{\mathbb{E}}-\mathbb{E}_{i}\right), \\
& \mathbf{N}_{i}^{\mathbf{k}} \cdot \mathbf{k}_{\infty}^{-1} \cdot\left(\overline{\mathrm{J}}-\mathrm{J}_{i}\right)=\left(\mathbf{N}_{i}^{\mathbf{k}}-\mathbf{I}\right) \cdot\left(\overline{\mathbb{K}}-\mathbb{K}_{i}\right),
\end{aligned}
$$

where $\mathbf{C}_{\infty}, \boldsymbol{\mu}_{\infty}, \boldsymbol{\epsilon}_{\infty}$, and $\mathbf{k}_{\infty}$ are the properties of the infinite medium in the inclusion problem and $\mathbf{N}_{i}^{\mathrm{C}}, \mathbf{N}_{i}^{\mu}, \mathbf{N}_{i}^{\epsilon}$, and $\mathbf{N}_{i}^{\mathbf{k}}$ are the corresponding depolarizing (or Eshelby) tensors (see Appendixes B and C). Adding the local coupling relations given by Eqs. (10), (14), (18), and (22) for each phase $i$, we obtain a system that can be solved in order to deduce the effective properties [Eq. (34)]. One should pay attention to the fact that the effective stiffness, permeability, permittivity, and thermal-conductivity tensors have different expressions than the ones defined in the-uncoupled problem [Eq. (37)]: for example, $\tilde{\boldsymbol{\mu}}$ links $\overline{\mathrm{B}}$ to $\overline{\mathbb{H}}$ when all the other total effective loadings are equal to zero. This permeability is not equal to $\bar{\mu}$ obtained when the coupled effects are locally canceled because of local incompatibilities due to the coupled behavior. 
TABLE I. Material properties (Ref. 4).

\begin{tabular}{lccc}
\hline \hline & & $\mathrm{BaTiO}_{3}$ & $\mathrm{CoFe}_{2} \mathrm{O}_{4}$ \\
\hline $\mathbf{C}_{11}$ & $(\mathrm{GPa})$ & 166 & 286 \\
$\mathbf{C}_{12}$ & & 77 & 173 \\
$\mathbf{C}_{13}$ & 78 & 170.5 \\
$\mathbf{C}_{33}$ & & 162 & 26.95 \\
$\mathbf{C}_{44} / 2$ & & 43 & 45.3 \\
$\boldsymbol{\mu}_{11}$ & $\left(10^{-6} \mathrm{~N} \mathrm{~s}^{2} / \mathrm{C}^{2}\right)$ & 5 & -590 \\
$\boldsymbol{\mu}_{33}$ & & 10 & 157 \\
$\boldsymbol{\epsilon}_{11}$ & $\left(10^{-10} \mathrm{C}^{2} / \mathrm{N} \mathrm{m}^{2}\right)$ & 112 & 0.8 \\
$\boldsymbol{\epsilon}_{33}$ & & 126 & 0.93 \\
$\mathbf{e}_{31}$ & $\left(\mathrm{C} / \mathrm{m}^{2}\right)$ & -4.4 & 0 \\
$\mathbf{e}_{33}$ & & 18.6 & 0 \\
$\mathbf{e}_{15} / \sqrt{2}$ & & 11.6 & 0 \\
$\mathbf{q}_{31}$ & $(\mathrm{~N} / \mathrm{A} \mathrm{m})$ & 0 & 580.3 \\
$\mathbf{q}_{33}$ & & 0 & 699.7 \\
$\mathbf{q}_{15} / \sqrt{2}$ & & 0 & 550 \\
\hline \hline
\end{tabular}

The general resolution scheme in the case of a fully coupled $n$-phasic material is detailed in Appendix E. A particular case of biphasic composite (for which localization rules can significantly be simplified) is given hereafter as an illustration.

\section{APPLICATION TO MAGNETOELECTRIC COMPOSITES}

The method is applied to a magnetoelectric composite made of piezomagnetic and piezoelectric materials. Composites made of magnetostrictive and electrostrictive phases exhibit a magnetoelectric effect. This effect is extrinsic since the coupling between electricity and magnetism appears through mechanics. Results in the literature show that the extrinsic magnetoelectric effect is larger in such composites than the intrinsic one ${ }^{19,20}$ observed in some homogeneous materials. We will use the configuration and material properties (see Table I) proposed by Li and Dunn, ${ }^{12}$ who proposed a homogenization model for those materials, and also taken by Lee et al. ${ }^{4}$ who compared these results to the corresponding finite element computations.

In the following, subscript $m$ stands for the piezomagnetic phase and subscript $e$ for the piezoelectric phase. The local constitutive laws are

$$
\left(\begin{array}{l}
\mathrm{T}_{m} \\
\mathbb{B}_{m} \\
\mathbb{D}_{m}
\end{array}\right)=\left(\begin{array}{ccc}
\mathbf{C}_{m} & -{ }^{t} \mathbf{q}_{m} & \mathbf{0} \\
\mathbf{q}_{m} & \boldsymbol{\mu}_{m} & \mathbf{0} \\
\mathbf{0} & \mathbf{0} & \boldsymbol{\epsilon}_{m}
\end{array}\right) \cdot\left(\begin{array}{l}
\mathrm{S}_{m} \\
\mathbb{H}_{m} \\
\mathbb{E}_{m}
\end{array}\right)
$$

for the piezomagnetic material $\left[\mathbf{q}_{m}\right.$ is the piezomagnetic matrix $(3 \times 6)]$ and

$$
\left(\begin{array}{c}
\mathrm{T}_{e} \\
\mathbb{B}_{e} \\
\mathbb{D}_{e}
\end{array}\right)=\left(\begin{array}{ccc}
\mathbf{C}_{e} & \mathbf{0} & -{ }^{t} \mathbf{e}_{e} \\
\mathbf{0} & \boldsymbol{\mu}_{e} & \mathbf{0} \\
\mathbf{e}_{e} & \mathbf{0} & \boldsymbol{\epsilon}_{e}
\end{array}\right) \cdot\left(\begin{array}{l}
\mathrm{S}_{e} \\
\mathrm{H}_{e} \\
\mathbb{E}_{e}
\end{array}\right)
$$

for the piezoelectric material $\left[\mathbf{e}_{e}\right.$ is the piezoelectric matrix $(3 \times 6)]$.
We apply the field decomposition,

$$
\begin{gathered}
\mathrm{S}_{m}=\mathrm{S}_{m}^{\mathbf{C}}+\mathrm{S}_{m}^{\boldsymbol{\mu}}, \\
\mathrm{H}_{m}=\mathrm{H}_{m}^{\boldsymbol{\mu}}+\mathbb{H}_{m}^{\mathbf{C}}, \\
\mathbb{E}_{m}=\mathbb{E}_{m}^{\boldsymbol{\epsilon}}, \\
\mathrm{S}_{e}=\mathrm{S}_{e}^{\mathbf{C}}+\mathrm{S}_{e}^{\boldsymbol{\epsilon}}, \\
\mathbb{H}_{e}=\mathbb{H}_{e}^{\boldsymbol{\mu}}, \\
\mathbb{E}_{e}=\mathbb{E}_{e}^{\boldsymbol{\epsilon}}+\mathbb{E}_{e}^{\mathbf{C}} .
\end{gathered}
$$

Then the homogenization equations can be written.

\section{A. Equations}

The mechanical system is

$$
\begin{aligned}
& \overline{\mathrm{S}}=\left\langle\mathrm{S}_{i}\right\rangle=f \cdot \mathrm{S}_{e}+(1-f) \cdot \mathrm{S}_{m}=f \cdot\left(\mathrm{S}_{e}^{\mathbf{C}}+\mathrm{S}_{e}^{\mathbf{\epsilon}}\right)+(1-f) \cdot\left(\mathrm{S}_{m}^{\mathbf{C}}\right. \\
& \left.+\mathrm{S}_{m}^{\mu}\right) \\
& \overline{\mathrm{S}}=\overline{\mathrm{S}} \mathbf{C}+\overline{\mathrm{S}}^{\boldsymbol{\epsilon}}+\overline{\mathrm{S}}^{\boldsymbol{\mu}}=\overline{\mathbf{C}}^{-1} \cdot \overline{\mathrm{T}}+\left\langle{ }^{t} \mathbf{B}_{i}^{\mathbf{C}} \cdot \mathrm{S}_{i}^{\boldsymbol{\epsilon}}\right\rangle+\left\langle{ }^{t} \mathbf{B}_{i}^{\mathbf{C}} \cdot \mathrm{S}_{i}^{\boldsymbol{\mu}}\right\rangle \\
& =\overline{\mathbf{C}}^{-1} \cdot \overline{\mathbb{T}}+f \cdot{ }^{t} \mathbf{B}_{e}^{\mathbf{C}} \cdot \mathrm{S}_{e}^{\boldsymbol{\epsilon}}+(1-f) \cdot{ }^{t} \mathbf{B}_{m}^{\mathbf{C}} \cdot \mathrm{S}_{m}^{\boldsymbol{\mu}}, \\
& \overline{\mathrm{T}}=\left\langle\mathrm{T}_{i}\right\rangle=\left\langle\mathbf{C}_{i} \cdot \mathrm{S}_{i}^{\mathbf{C}}\right\rangle \\
& =f \cdot \mathbf{C}_{e} \cdot \mathrm{S}_{e}^{\mathbf{C}}+(1-f) \cdot \mathbf{C}_{m} \cdot \mathrm{S}_{m}^{\mathbf{C}},
\end{aligned}
$$

with $f$ the volumetric fraction of the piezoelectric phase. Similarly, the magnetic system can be written as

$$
\begin{gathered}
\overline{\mathrm{H}}=\left\langle\mathrm{H}_{i}\right\rangle=f \cdot \mathrm{H}_{e}+(1-f) \cdot \mathrm{H}_{m}=f \cdot \mathbb{H}_{e}^{\boldsymbol{\mu}}+(1-f) \cdot\left(\mathrm{H}_{m}^{\boldsymbol{\mu}}+\mathbb{H}_{m}^{\mathbf{C}}\right), \\
\overline{\mathrm{H}}=\overline{\mathrm{H}}^{\boldsymbol{\mu}}+\overline{\mathbb{H}^{\mathbf{C}}}=\overline{\boldsymbol{\mu}}^{-1} \cdot \overline{\mathrm{B}}+\left\langle{ }^{t} \mathbf{B}_{i}^{\boldsymbol{\mu}} \cdot \mathrm{H}_{i}^{\mathbf{C}}\right\rangle \\
=\overline{\boldsymbol{\mu}}^{-1} \cdot \overline{\mathrm{B}}+(1-f) \cdot{ }^{t} \mathbf{B}_{m}^{\boldsymbol{\mu}} \cdot \mathrm{H}_{m}^{\mathbf{C}}, \\
\overline{\mathrm{B}}=\left\langle\mathrm{B}_{i}\right\rangle=\left\langle\boldsymbol{\mu}_{i} \cdot \mathbb{H}_{i}^{\boldsymbol{\mu}}\right\rangle=f \cdot \boldsymbol{\mu}_{e} \cdot \mathrm{H}_{e}^{\boldsymbol{\mu}}=(1-f) \cdot \boldsymbol{\mu}_{m} \cdot \mathbb{H}_{m}^{\boldsymbol{\mu}} .
\end{gathered}
$$

Finally, the electric system can be written as

$$
\begin{gathered}
\overline{\mathbb{E}}=\left\langle\mathbb{E}_{i}\right\rangle=f \cdot \mathbb{E}_{e}+(1-f) \cdot \mathbb{E}_{m}=f \cdot\left(\mathbb{E}_{e}^{\boldsymbol{\epsilon}}+\mathbb{E}_{e}^{\mathbf{C}}\right)+(1-f) \cdot \mathbb{E}_{m}^{\boldsymbol{\epsilon}}, \\
\overline{\mathbb{E}}=\overline{\mathbb{E}}{ }^{\boldsymbol{\epsilon}}+\overline{\mathbb{E}}^{\mathbf{C}}=\overline{\boldsymbol{\epsilon}}^{-1} \cdot \overline{\mathrm{D}}+\left\langle{ }^{t} \mathbf{B}_{i}^{\boldsymbol{\epsilon}} \cdot \mathbb{E}_{i}^{\mathbf{C}}\right\rangle=\overline{\boldsymbol{\epsilon}}^{-1} \cdot \overline{\mathrm{D}}+f \cdot{ }^{t} \mathbf{B}_{i}^{\boldsymbol{\epsilon}} \cdot \mathbb{E}_{e}^{\mathbf{C}}, \\
\overline{\mathrm{D}}=\left\langle\mathrm{D}_{i}\right\rangle=\left\langle\boldsymbol{\epsilon}_{i} \cdot \mathbb{E}_{i}^{\boldsymbol{\epsilon}}\right\rangle=f \cdot \boldsymbol{\epsilon}_{e} \cdot \mathbb{E}_{e}^{\boldsymbol{\epsilon}}+(1-f) \cdot \boldsymbol{\epsilon}_{m} \cdot \mathbb{E}_{m}^{\boldsymbol{\epsilon}} .
\end{gathered}
$$

Moreover, The coupling relations are given by Eqs. (54) and (55), respectively, for the piezomagnetic and piezoelectric phases,

$$
\begin{gathered}
-\mathbf{C}_{m} \cdot \mathrm{S}_{m}^{\boldsymbol{\mu}}=-{ }^{t} \mathbf{q}_{m} \cdot \mathbb{H}_{m}=-{ }^{t} \mathbf{q}_{m} \cdot\left(\mathbb{H}_{m}^{\boldsymbol{\mu}}+\mathbb{H}_{m}^{\mathbf{C}}\right), \\
-\boldsymbol{\mu}_{m} \cdot \mathrm{H}_{m}^{\mathbf{C}}=\mathbf{q}_{m} \cdot \mathrm{S}_{m}=\mathbf{q}_{m} \cdot\left(\mathrm{S}_{m}^{\mathbf{C}}+\mathrm{S}_{m}^{\boldsymbol{\mu}}\right), \\
-\mathbf{C}_{e} \cdot \mathrm{S}_{e}^{\boldsymbol{\epsilon}}=-{ }^{t} \mathbf{e}_{e} \cdot \mathbb{E}_{e}=-{ }^{t} \mathbf{e}_{e} \cdot\left(\mathbb{E}_{e}^{\boldsymbol{\epsilon}}+\mathbb{E}_{e}^{\mathbf{C}}\right), \\
-\boldsymbol{\epsilon}_{e} \cdot \mathbb{E}_{e}^{\mathbf{C}}=\mathbf{e}_{e} \cdot \mathrm{S}_{e}=\mathbf{e}_{e} \cdot\left(\mathrm{S}_{e}^{\mathbf{C}}+\mathrm{S}_{e}^{\boldsymbol{\epsilon}}\right) .
\end{gathered}
$$






FIG. 3. Modeling scheme.

\section{B. Modeling scheme}

The system is constituted of 16 unknowns: (i) the macroscopic loading $\overline{\mathrm{S}}, \overline{\mathrm{H}}$, and $\overline{\mathbb{E}}$; (ii) the macroscopic response $\overline{\mathbb{T}}$, $\overline{\mathrm{B}}$, and $\overline{\mathrm{D}}$; (iii) the local decomposed strains $\mathrm{S}_{e}^{\mathbf{C}}, \mathrm{S}_{m}^{\mathbf{C}}, \mathrm{S}_{e}^{\boldsymbol{\epsilon}}$, and $\mathrm{S}_{m}^{\mu}$; (iv) the local decomposed magnetic fields $\mathbb{H}_{e}^{\mu}, \mathbb{H}_{m}^{\mu}$, and $\mathrm{H}_{m}^{\mathbf{C}} ;(\mathrm{v})$ and the local decomposed electric fields $\mathbb{E}_{e}^{\boldsymbol{\epsilon}}, \mathbb{E}_{m}^{\boldsymbol{\epsilon}}$, and $\mathbb{E}_{e}^{C}$.

These 16 unknowns are linked through 13 equations (three from mechanics [Eq. (51)], three from magnetism [Eq. (52)], three from electricity [Eq. (53)], two from piezomagnetism [Eq. (54)], and two from piezoelectricity [Eq. (55)]).

The effective properties of the composite can be deduced eliminating the local fields. Then the system reduces to three equations with six unknowns (the macroscopic fields) (Fig. 3 ). For a given applied loading (three parameters), the macroscopic response is given by the constitutive law [Eq. (56)]. If needed, the local fields and corresponding decomposition can also be calculated,

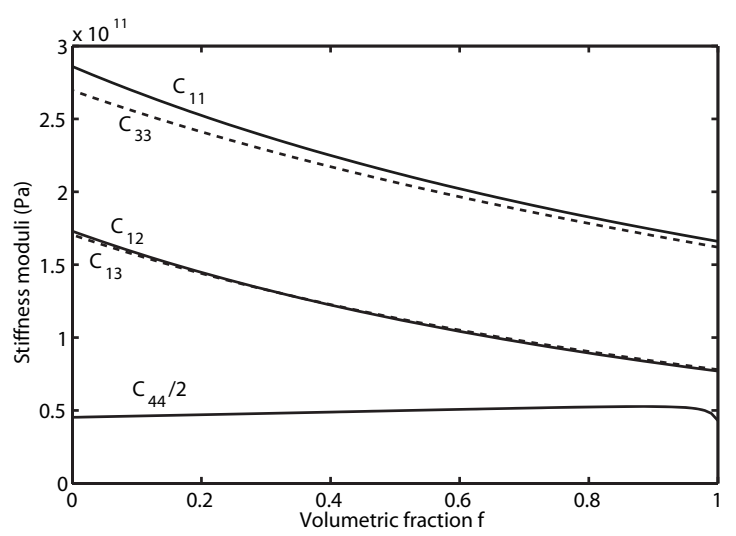

FIG. 4. Effective stiffness moduli depending on the volumetric fraction $f$ of the piezoelectric phase.

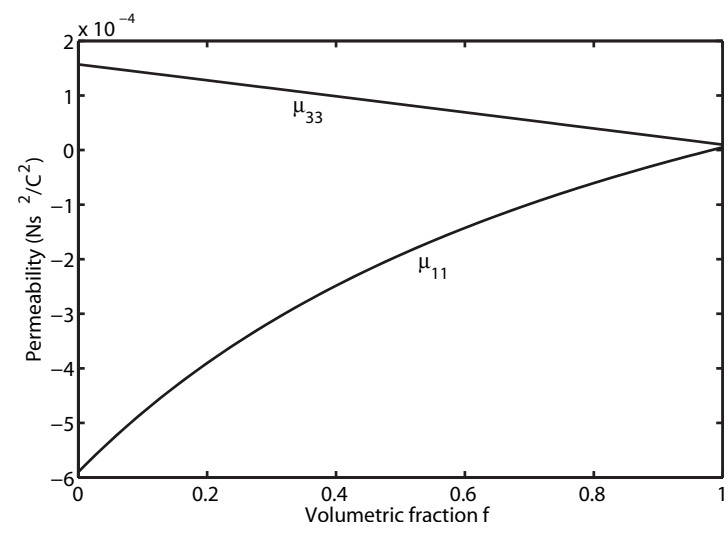

FIG. 5. Effective permeability depending on the volumetric fraction $f$ of the piezoelectric phase.

$$
\left(\begin{array}{c}
\bar{T} \\
\overline{\mathbb{B}} \\
\overline{\mathrm{D}}
\end{array}\right)=\left(\begin{array}{ccc}
\widetilde{\mathbf{C}} & -{ }^{t} \widetilde{\mathbf{q}} & -{ }^{t} \widetilde{\mathbf{e}} \\
\widetilde{\mathbf{q}} & \tilde{\boldsymbol{\mu}} & { }^{t} \widetilde{\boldsymbol{\alpha}} \\
\widetilde{\mathbf{e}} & \tilde{\boldsymbol{\alpha}} & \tilde{\boldsymbol{\epsilon}}
\end{array}\right) \cdot\left(\begin{array}{c}
\overline{\mathrm{S}} \\
\overline{\mathrm{H}} \\
\overline{\mathbb{E}}
\end{array}\right) .
$$

\section{Results}

The modeled composite is a piezomagnetic matrix $\left(\mathrm{CoFe}_{2} \mathrm{O}_{4}\right)$ reinforced with cylindrical piezoelectric fibers $\left(\mathrm{BaTiO}_{3}\right)$. For such a matrix and fibers microstructure, an appropriate choice for the infinite medium properties in the homogenization scheme is the properties of the matrix. This choice will correspond to a Mori-Tanaka-type estimate. ${ }^{17}$ Some complementary elements to calculate the localization tensors in the case of infinite cylindrical inclusions are given in Appendix $\mathrm{C}$. The volumetric fraction of the piezoelectric phase is $f$. The effective properties [Eq. (56)] obtained with the proposed homogenization model are shown in the following figures (Fig. 4-9).

These modeling results are the same than the ones obtained by Li and Dunn. ${ }^{12}$ Their model is based upon a modified localization tensor for coupled properties. These results are also in good agreement with the ones obtained by Lee et $a l .{ }^{4}$ with a finite element modeling.

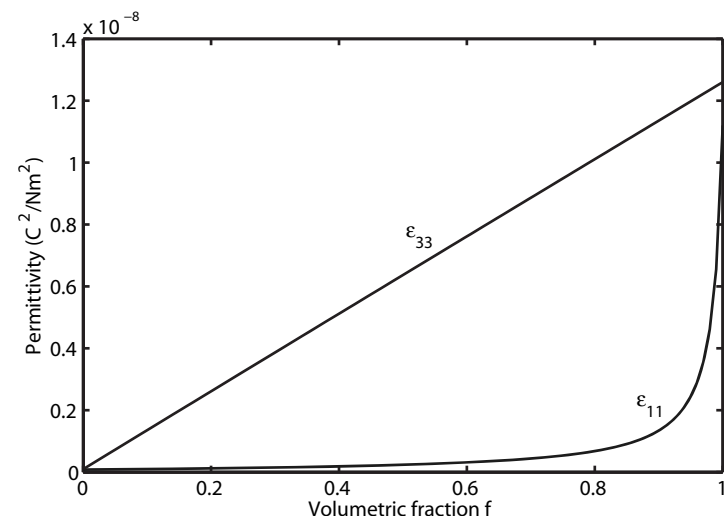

FIG. 6. Effective permittivity depending on the volumetric fraction $f$ of the piezoelectric phase. 


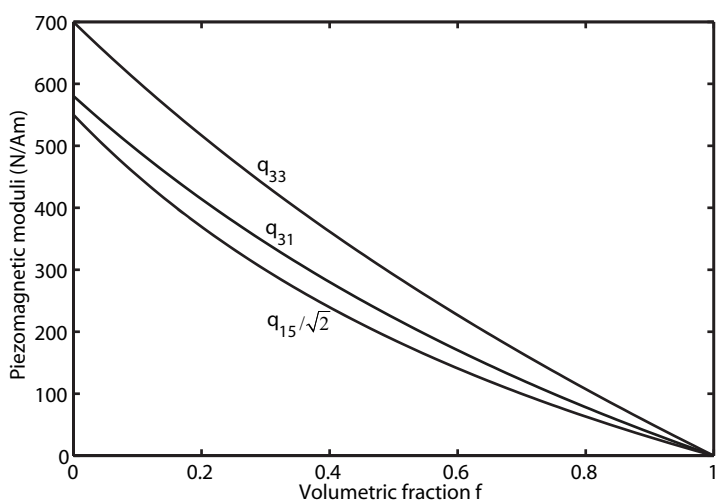

FIG. 7. Effective piezomagnetic moduli depending on the volumetric fraction $f$ of the piezoelectric phase.

It is very noticeable that the effective properties of the composite made of piezomagnetic and piezoelectric materials exhibit a magnetoelectric effect, whereas none of the phases exhibit such an effect locally. This magnetoelectric effect in composites is typically a microstructure effect. In that sense it is particularly suited to validate homogenization tools.

\section{CONCLUSION}

We proposed a generic formalism for the homogenization of coupled behavior. It is based on the decomposition of the state variables into several contributions representing the coupling effects. That way, the classical rules of homogenization for uncoupled behavior can be maintained. They have to be completed by some additional coupling rules. This approach gives identical macroscopic results in the case of magnetoelectroelastic behavior with some previous results taken in the literature. The advantages of the proposed method are numerous: it applies to any kind of coupling among magnetoelectrothermoelastic couplings; it applies to any composite material, whatever the number of phases, and even for polycrystals (each phase being associated to a particular crystallographic direction); different types of microstructure are accounted for, through the choice of the properties of the infinite medium in the inclusion problem; it allows us to define the values of the local and macroscopic



FIG. 8. Effective piezoelectric moduli depending on the volumetric fraction $f$ of the piezoelectric phase.

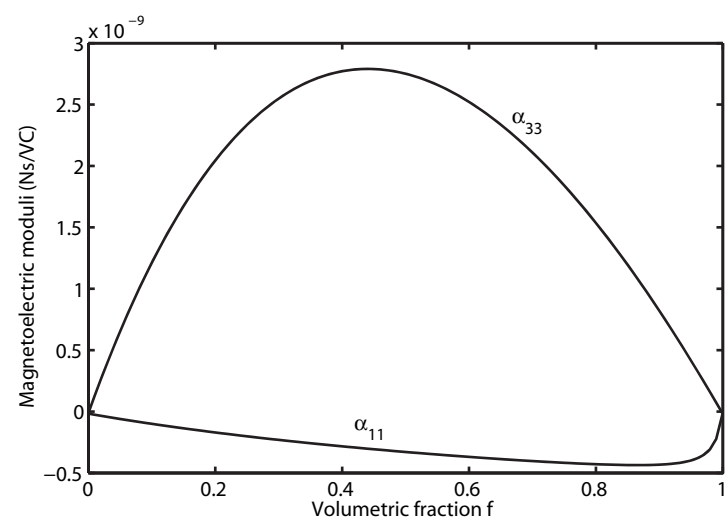

FIG. 9. Effective magnetoelectric moduli depending on the volumetric fraction $f$ of the piezoelectric phase.

contributions of each coupling effect on the overall response of the material; and it does not introduce any ambiguousness concerning the properties linking the dual state variables (elastic stiffness, magnetic permeability, and electric permittivity and thermal-conductivity tensors) that are defined in a unique way.

The proposed approach considers linear behavior. Many smart materials exhibit nonlinear behavior; but in many cases, the linearization of the behavior around the operating point gives satisfying approximation. In the case when this nonlinearity cannot be neglected, for example, for devices used in a wide range of loadings, an extension of this method has to be achieved. This point is currently a work in progress. To conclude, we can emphasize the fact that the proposed decomposition for the state variables (already used in mechanics when dealing with thermal strain or plasticity) can be used in other context than homogenization and could give interesting results in other modeling techniques such as finite element methods.

\section{APPENDIX A: MODIFIED VOIGT NOTATION}

When second-, third-, and fourth-order tensors exhibit some symmetries, the modified Voigt notation (also named Voigt-Mandel notation) can be used in order to manipulate only matrices. For a second-order tensor $\mathbb{X}$ with symmetries so that $X_{i j}=X_{j i}$, the modified Voigt notation gives the following representation $(6 \times 1)$ :

$$
\mathbb{X}=\left(\begin{array}{ccc}
X_{11} & X_{12} & X_{13} \\
X_{21} & X_{22} & X_{23} \\
X_{31} & X_{32} & X_{33}
\end{array}\right) \rightarrow\left(\begin{array}{c}
X_{11} \\
X_{22} \\
X_{33} \\
\sqrt{2} X_{23} \\
\sqrt{2} X_{31} \\
\sqrt{2} X_{12}
\end{array}\right) .
$$

Similarly, a fourth-order tensor $\mathbf{F}$ exhibiting the following symmetries: $F_{i j k l}=F_{j i k l}=F_{i j l k}=F_{j i l k}$ can be written using modified Voigt notation. The $(6 \times 6)$ representation is given by Eq. (A2) 


$$
\mathbf{F}=\left(F_{i j k l}\right) \rightarrow\left(\begin{array}{cccccc}
F_{1111} & F_{1122} & F_{1133} & \sqrt{2} F_{1123} & \sqrt{2} F_{1131} & \sqrt{2} F_{1112} \\
F_{2211} & F_{2222} & F_{2233} & \sqrt{2} F_{2223} & \sqrt{2} F_{2231} & \sqrt{2} F_{2212} \\
F_{3311} & F_{3322} & F_{3333} & \sqrt{2} F_{3323} & \sqrt{2} F_{3331} & \sqrt{2} F_{3312} \\
\sqrt{2} F_{2311} & \sqrt{2} F_{2322} & \sqrt{2} F_{2333} & 2 F_{2323} & 2 F_{2331} & 2 F_{2312} \\
\sqrt{2} F_{3111} & \sqrt{2} F_{3122} & \sqrt{2} F_{3133} & 2 F_{3123} & 2 F_{3131} & 2 F_{3112} \\
\sqrt{2} F_{1211} & \sqrt{2} F_{1222} & \sqrt{2} F_{1233} & 2 F_{1223} & 2 F_{1231} & 2 F_{1212}
\end{array}\right) .
$$

For a third-order tensor $\mathbf{d}$ exhibiting symmetries $d_{i j k}=d_{i k j}$, the $(3 \times 6)$ representation is used [Eq. (A3)],

$$
\mathbf{d}=\left(d_{i j k}\right) \rightarrow\left(\begin{array}{llllll}
d_{111} & d_{122} & d_{133} & \sqrt{2} d_{123} & \sqrt{2} d_{131} & \sqrt{2} d_{112} \\
d_{211} & d_{222} & d_{233} & \sqrt{2} d_{223} & \sqrt{2} d_{231} & \sqrt{2} d_{212} \\
d_{311} & d_{322} & d_{333} & \sqrt{2} d_{323} & \sqrt{2} d_{331} & \sqrt{2} d_{312}
\end{array}\right) .
$$

\section{APPENDIX B: DEFINITION OF LOCALIZATION TENSORS}

The basic-uncoupled-inclusion problem consists of an inclusion $i$ embedded in an infinite medium $\infty$ with a uniform applied loading at the infinity. When the shape of the inclusion is ellipsoidal, then the field in the inclusion is uniform. ${ }^{16}$ In a mechanical problem, noting $\mathbf{C}_{i}$ the stiffness tensor of the inclusion, $\mathbf{C}_{\infty}$ the stiffness tensor of the infinite medium, and $\mathrm{S}_{\infty}$ the uniform applied loading at the infinity, the (uniform) strain in the inclusion is given by

$$
\mathrm{S}_{i}=\left[\mathbf{I}+\mathbf{N}_{i}^{\mathbf{C}} \cdot \mathbf{C}_{\infty}^{-1} \cdot\left(\mathbf{C}_{i}-\mathbf{C}_{\infty}\right)\right]^{-1} \cdot \mathrm{S}_{\infty},
$$

where $\mathbf{N}_{i}^{\mathbf{C}}$ is the Eshelby tensor $(6 \times 6)$ depending on the shape of the inclusion and on the properties of the infinite medium (details to calculate the Eshelby tensor can be found in Mura's ${ }^{21}$ monograph).

Homogenization techniques based on inclusion problems divide the $n$-phasic problem into $n$ uncorrelated basic inclusion problems. The uniform applied loading at the infinity $\mathrm{S}_{\infty}$ in the basic inclusion problem is not directly the macroscopic loading ${ }^{18} \overline{\mathrm{S}}$. Indeed, the local strains must verify $\left\langle\mathrm{S}_{i}\right\rangle=\overline{\mathrm{S}}$. Then, the-uncoupled-mechanical localization tensor $\mathbf{A}_{i}^{\mathbf{C}}\left(\mathrm{S}_{i}\right.$ $\left.=\mathbf{A}_{i}^{\mathbf{C}} \cdot \overline{\mathrm{S}}\right)$ can be deduced as

$$
\begin{aligned}
\mathbf{A}_{i}^{\mathbf{C}}= & {\left[\mathbf{I}+\mathbf{N}_{i}^{\mathbf{C}} \cdot \mathbf{C}_{\infty}^{-1} \cdot\left(\mathbf{C}_{i}-\mathbf{C}_{\infty}\right)\right]^{-1} \cdot\left\langle\left[\mathbf{I}+\mathbf{N}_{i}^{\mathbf{C}} \cdot \mathbf{C}_{\infty}^{-1} \cdot\left(\mathbf{C}_{i}\right.\right.\right.} \\
& \left.\left.\left.-\mathbf{C}_{\infty}\right)\right]^{-1}\right\rangle^{-1} .
\end{aligned}
$$

The same way, the-uncoupled-magnetic localization tensor $\mathbf{A}_{i}^{\mu}\left(\mathbb{H}_{i}=\mathbf{A}_{i}^{\mu} \cdot \mathbb{H}\right)$ can be obtained as

$$
\begin{aligned}
\mathbf{A}_{i}^{\boldsymbol{\mu}}= & {\left[\mathbf{I}+\mathbf{N}_{i}^{\boldsymbol{\mu}} \cdot \boldsymbol{\mu}_{\infty}^{-1} \cdot\left(\boldsymbol{\mu}_{i}-\boldsymbol{\mu}_{\infty}\right)\right]^{-1} \cdot\left\langle\left[\mathbf{I}+\mathbf{N}_{i}^{\boldsymbol{\mu}} \cdot \boldsymbol{\mu}_{\infty}^{-1} \cdot\left(\boldsymbol{\mu}_{i}\right.\right.\right.} \\
& \left.\left.\left.-\boldsymbol{\mu}_{\infty}\right)\right]^{-1}\right\rangle^{-1},
\end{aligned}
$$

where $\mathbf{N}_{i}^{\mu}$ is the demagnetizing tensor $(3 \times 3)$ (equivalent to the Eshelby tensor in mechanics).

Similarly, the-uncoupled-electric localization tensor $\mathbf{A}_{i}^{\boldsymbol{\epsilon}}$ $\left(\mathbb{E}_{i}=\mathbf{A}_{i}^{\epsilon} \cdot \overline{\mathbb{E}}\right)$ can be obtained as

$$
\mathbf{A}_{i}^{\boldsymbol{\epsilon}}=\left[\mathbf{I}+\mathbf{N}_{i}^{\boldsymbol{\epsilon}} \cdot \boldsymbol{\epsilon}_{\infty}^{-1} \cdot\left(\boldsymbol{\epsilon}_{i}-\boldsymbol{\epsilon}_{\infty}\right)\right]^{-1} \cdot\left\langle\left[\mathbf{I}+\mathbf{N}_{i}^{\boldsymbol{\epsilon}} \cdot \boldsymbol{\epsilon}_{\infty}^{-1} \cdot\left(\boldsymbol{\epsilon}_{i}-\boldsymbol{\epsilon}_{\infty}\right)\right]^{-1}\right\rangle^{-1}
$$

with $\mathbf{N}_{i}^{\epsilon}$ the depolarizing tensor in the electric problem. Theuncoupled-thermal localization tensor $\mathbf{A}_{i}^{\mathbf{k}}\left(\mathbb{K}_{i}=\mathbf{A}_{i}^{\mathbf{k}} \cdot \overline{\mathbb{K}}\right)$ can be obtained as

$$
\begin{aligned}
\mathbf{A}_{i}^{\mathbf{k}}= & {\left[\mathbf{I}+\mathbf{N}_{i}^{\mathbf{k}} \cdot \mathbf{k}_{\infty}^{-1} \cdot\left(\mathbf{k}_{i}-\mathbf{k}_{\infty}\right)\right]^{-1} \cdot\left\langle\left[\mathbf{I}+\mathbf{N}_{i}^{\mathbf{k}} \cdot \mathbf{k}_{\infty}^{-1} \cdot\left(\mathbf{k}_{i}\right.\right.\right.} \\
& \left.\left.\left.-\mathbf{k}_{\infty}\right)\right]^{-1}\right\rangle^{-1}
\end{aligned}
$$

with $\mathbf{N}_{i}^{\mathbf{k}}$ the depolarizing tensor as the thermal problem.

The choice of the infinite medium properties gives a degree of freedom for the construction of several homogenization models. This choice is directly connected to the type of microstructure of the real heterogeneous material. When the microstructure consists of inclusions embedded in a homogeneous matrix, then an appropriate choice for the infinite medium properties is the matrix properties (corresponding to the Mori-Tanaka estimate in mechanics).

\section{APPENDIX C: ESHELBY AND DEPOLARIZING TENSORS IN THE CYLINDRICAL CASE}

For an infinite cylindrical inclusion $i$ (embedded in an infinite medium $\infty$ with transversely isotropic stiffness tensor $\mathbf{C}_{\infty}$ ), the Eshelby tensor $\mathbf{N}_{i}^{\mathbf{C}}$ is given by (cylinder along the axis 3)

$$
\begin{gathered}
\mathbf{N}_{i 11}^{\mathbf{C}}=\mathbf{N}_{i 22}^{\mathbf{C}}=\frac{5 \mathbf{C}_{\infty 11}+\mathbf{C}_{\infty 12}}{8 \mathbf{C}_{\infty 11}}, \\
\mathbf{N}_{i 12}^{\mathbf{C}}=\mathbf{N}_{i 21}^{\mathbf{C}}=\frac{-\mathbf{C}_{\infty 11}+3 \mathbf{C}_{\infty 12}}{8 \mathbf{C}_{\infty 11}}, \\
\mathbf{N}_{i 13}^{\mathbf{C}}=\mathbf{N}_{i 23}^{\mathbf{C}}=\frac{\mathbf{C}_{\infty 13}}{2 \mathbf{C}_{\infty 11}}, \\
\mathbf{N}_{i 44}^{\mathbf{C}}=\mathbf{N}_{i 55}^{\mathbf{C}}=\frac{1}{2}, \\
\mathbf{N}_{i 66}^{\mathbf{C}}=\frac{3 \mathbf{C}_{\infty 11}-\mathbf{C}_{\infty 12}}{4 \mathbf{C}_{\infty 11}} .
\end{gathered}
$$

All others terms are null.

In the case of diagonal permeability, permittivity, or thermal-conductivity tensors, the depolarizing tensors only 
depend on the shape of the inclusion. For a cylindrical inclusion (along axis 3 ),

$$
\mathbf{N}_{i}^{\mu}=\mathbf{N}_{i}^{\epsilon}=\mathbf{N}_{i}^{\mathbf{k}}=\left(\begin{array}{ccc}
1 / 2 & 0 & 0 \\
0 & 1 / 2 & 0 \\
0 & 0 & 0
\end{array}\right) .
$$

\section{APPENDIX D: DECOMPOSITION OF THE MACROSCOPIC FIELDS}

The following proof is given in mechanics ${ }^{22}$ but it can be generalized to magnetism, electricity, and thermics:

$$
\left\langle{ }^{t} \mathbf{B}_{i}^{\mathbf{C}} \cdot \mathrm{S}_{i}\right\rangle=\left\langle{ }^{t} \mathbf{B}_{i}^{\mathbf{C}} \cdot \mathrm{S}_{i}^{\mathbf{C}}\right\rangle+\left\langle{ }^{t} \mathbf{B}_{i}^{\mathbf{C}} \cdot\left(\mathrm{S}_{i}^{\boldsymbol{\mu}}+\mathrm{S}_{i}^{\boldsymbol{\epsilon}}+\mathrm{S}_{i}^{\mathbf{k}}\right)\right\rangle .
$$

Using Eq. (8) leads to

$$
\left\langle{ }^{t} \mathbf{B}_{i}^{\mathbf{C}} \cdot \mathrm{S}_{i}^{\mathbf{C}}\right\rangle=\left\langle{ }^{t} \mathbf{B}_{i}^{\mathbf{C}} \cdot \mathbf{C}_{i}^{-1} \cdot \mathbb{T}_{i}\right\rangle,
$$

which is equal to

$$
\left\langle{ }^{t} \mathbf{B}_{i}^{\mathbf{C}} \cdot \mathrm{S}_{i}^{\mathbf{C}}\right\rangle={ }^{t}\left\langle\mathrm{~T}_{i} \cdot \mathbf{C}_{i}^{-1} \cdot \mathbf{B}_{i}^{\mathbf{C}}\right\rangle
$$

because $\mathbb{T}_{i}$ and $\mathbf{C}_{i}$ are symmetric tensors.

Moreover, the Hill theorem ${ }^{22}$ enables us to write

$$
\left\langle\mathbb{T}_{i} \cdot \mathbf{C}_{i}^{-1} \cdot \mathbf{B}_{i}^{\mathbf{C}}\right\rangle=\left\langle\mathbb{T}_{i}\right\rangle \cdot\left\langle\mathbf{C}_{i}^{-1} \cdot \mathbf{B}_{i}^{\mathbf{C}}\right\rangle=\overline{\mathbb{T}} \cdot \overline{\mathbf{C}}^{-1}
$$

with $\overline{\mathbf{C}}$ as the effective stiffness tensor introduced in the purely mechanical problem [Eq. (40)]. Then coming back to Eq. (D3), we obtain

$$
\left\langle{ }^{t} \mathbf{B}_{i}^{\mathbf{C}} \cdot \mathrm{S}_{i}^{\mathbf{C}}\right\rangle={ }^{t}\left(\overline{\mathrm{T}} \cdot \overline{\mathbf{C}}^{-1}\right)=\overline{\mathbf{C}}^{-1} \cdot \overline{\mathrm{T}}
$$

because $\overline{\mathbb{T}}$ and $\overline{\mathbf{C}}$ are symmetric tensors. Additionnaly, $\overline{\mathbf{C}}^{-1} \cdot \overline{\mathrm{T}}$ is equal to $\overline{S^{C}}$ according to Eq. (41). We can conclude that $\left\langle{ }^{t} \mathbf{B}_{i}^{\mathbf{C}} \cdot \mathrm{S}_{i}^{\mathbf{C}}\right\rangle=\overline{\mathrm{S}} \mathbf{C}$.

Moreover, the first equation also verifies

$$
\left\langle{ }^{t} \mathbf{B}_{i}^{\mathbf{C}} \cdot \mathrm{S}_{i}\right\rangle=\left\langle{ }^{t} \mathbf{B}_{i}^{\mathbf{C}}\right\rangle \cdot\left\langle\mathrm{S}_{i}\right\rangle=\overline{\mathrm{S}}
$$

because of the Hill theorem. Finally, the following equalities are obtained:

$$
\begin{gathered}
\overline{\mathrm{S}}=\overline{\mathrm{S}}^{\mathbf{C}}+\overline{\mathrm{S}}^{\boldsymbol{\mu}}+\overline{\mathrm{S}}^{\boldsymbol{\epsilon}}+\overline{\mathrm{S}}^{\mathbf{k}}, \\
\overline{\mathrm{S}}^{\mathbf{C}}=\left\langle{ }^{t} \mathbf{B}_{i}^{\mathbf{C}} \cdot \mathrm{S}_{i}^{\mathbf{C}}\right\rangle=\overline{\mathbf{C}}^{-1} \cdot \overline{\mathrm{T}} \neq\left\langle\mathrm{S}_{i}^{\mathbf{C}}\right\rangle, \\
\overline{\mathrm{S}}^{\boldsymbol{\mu}}=\left\langle{ }^{t} \mathbf{B}_{i}^{\mathbf{C}} \cdot \mathrm{S}_{i}^{\boldsymbol{\mu}}\right\rangle \neq\left\langle\mathrm{S}_{i}^{\boldsymbol{\mu}}\right\rangle, \\
\overline{\mathrm{S}}^{\boldsymbol{\epsilon}}=\left\langle{ }^{t} \mathbf{B}_{i}^{\mathbf{C}} \cdot \mathrm{S}_{i}^{\boldsymbol{\epsilon}}\right\rangle \neq\left\langle\mathrm{S}_{i}^{\boldsymbol{\epsilon}}\right\rangle,
\end{gathered}
$$

$$
\overline{\mathrm{S}}^{\mathbf{k}}=\left\langle{ }^{t} \mathbf{B}_{i}^{\mathbf{C}} \cdot \mathrm{S}_{i}^{\mathbf{k}}\right\rangle \neq\left\langle\mathrm{S}_{i}^{\mathbf{k}}\right\rangle .
$$

\section{APPENDIX E: GENERAL RESOLUTION SCHEME}

We define hereafter the system of equations to be solved in the general case of a fully coupled $n$-phasic composite. ${ }^{23}$ The set of unknowns consists of eight macroscopic (total) fields,

$$
\begin{aligned}
& \overline{\mathrm{T}}, \quad \overline{\mathrm{B}}, \quad \overline{\mathrm{D}}, \quad \overline{\mathrm{J}}, \\
& \overline{\mathrm{S}}, \quad \overline{\mathrm{H}}, \quad \overline{\mathrm{E}}, \quad \overline{\mathrm{K}},
\end{aligned}
$$

$8 n$ local (total) fields,

$$
\begin{aligned}
& \mathrm{T}_{i}, \quad \mathrm{~B}_{i}, \quad \mathrm{D}_{i}, \quad \mathrm{~J}_{i}, \\
& \mathrm{~S}_{i}, \quad \mathbb{H}_{i}, \quad \mathbb{E}_{i}, \quad \mathbb{K}_{i},
\end{aligned}
$$

$16 n$ local decomposed fields,

$$
\begin{array}{cccc}
\mathrm{S}_{i}^{\mathbf{C}}, & \mathbb{H}_{i}^{\mathbf{C}}, & \mathbb{E}_{i}^{\mathbf{C}}, & \mathbb{K}_{i}^{\mathbf{C}}, \\
\mathrm{S}_{i}^{\boldsymbol{\mu}}, & \mathbb{H}_{i}^{\boldsymbol{\mu},}, & \mathbb{E}_{i}^{\boldsymbol{\mu}}, & \mathbb{K}_{i}^{\boldsymbol{\mu}}, \\
\mathrm{S}_{i}^{\boldsymbol{\epsilon}}, & \mathrm{H}_{i}^{\boldsymbol{\epsilon}}, & \mathbb{E}_{i}^{\boldsymbol{\epsilon}}, & \mathbb{K}_{i}^{\boldsymbol{\epsilon}}, \\
\mathrm{S}_{i}^{\mathbf{k}}, & \mathbb{H}_{i}^{\mathbf{k}}, & \mathbb{E}_{i}^{\mathbf{k}}, & \mathbb{K}_{i}^{\mathbf{k}} .
\end{array}
$$

The system of equations is given by (i) macroscopic applied loadings: four equations involving the macroscopic (total) fields, (ii) macroscopic decomposition of the fields: 4 equations [obtained from Eqs. (42)-(45)],

$$
\begin{aligned}
& \overline{\mathrm{S}}=\overline{\mathbf{C}}^{-1} \cdot \overline{\mathbb{T}}+\left\langle{ }^{t} \mathbf{B}_{i}^{\mathbf{C}} \cdot \mathrm{S}_{i}^{\boldsymbol{\mu}}\right\rangle+\left\langle{ }^{t} \mathbf{B}_{i}^{\mathbf{C}} \cdot \mathrm{S}_{i}^{\boldsymbol{\epsilon}}\right\rangle+\left\langle{ }^{t} \mathbf{B}_{i}^{\mathbf{C}} \cdot \mathrm{S}_{i}^{\mathbf{k}}\right\rangle, \\
& \overline{\mathbb{H}}=\left\langle{ }^{t} \mathbf{B}_{i}^{\boldsymbol{\mu}} \cdot \mathbb{H}_{i}^{\mathbf{C}}\right\rangle+\overline{\boldsymbol{\mu}}^{-1} \overline{\mathrm{B}}+\left\langle{ }^{t} \mathbf{B}_{i}^{\boldsymbol{\mu}} \cdot \mathbb{H}_{i}^{\boldsymbol{\epsilon}}\right\rangle+\left\langle{ }^{t} \mathbf{B}_{i}^{\mu} \cdot \mathbb{H}_{i}^{\mathbf{k}}\right\rangle, \\
& \overline{\mathbb{E}}=\left\langle{ }^{t} \mathbf{B}_{i}^{\boldsymbol{\epsilon}} \cdot \mathbb{E}_{i}^{\mathbf{C}}\right\rangle+\left\langle{ }^{t} \mathbf{B}_{i}^{\boldsymbol{\epsilon}} \cdot \mathbb{E}_{i}^{\boldsymbol{\mu}}\right\rangle+\overline{\boldsymbol{\epsilon}}^{-1} \overline{\mathrm{D}}+\left\langle{ }^{t} \mathbf{B}_{i}^{\boldsymbol{\epsilon}} \cdot \mathbb{E}_{i}^{\mathbf{k}}\right\rangle, \\
& \overline{\mathbb{K}}=\left\langle{ }^{t} \mathbf{B}_{i}^{\mathbf{k}} \cdot \mathbb{K}_{i}^{\mathbf{C}}\right\rangle+\left\langle{ }^{t} \mathbf{B}_{i}^{\mathbf{k}} \cdot \mathbb{K}_{i}^{\mu}\right\rangle+\left\langle{ }^{t} \mathbf{B}_{i}^{\mathbf{k}} \cdot \mathbb{K}_{i}^{\boldsymbol{\epsilon}}\right\rangle+\overline{\mathbf{k}}^{-1} \overline{\mathrm{J}},
\end{aligned}
$$

(iii) local decomposition of the fields: $4 n$ equations [Eqs. (7), (11), (15), and (19)], uncoupled local constitutive laws: $4 n$ equations [Eqs. (8), (12), (16), and (20)], (iv) local coupling relations: $12 n$ equations [Eqs. (10), (14), (18), and (22)], (v) localization relations: $4 n$ equations [Eq. (46)]. We then obtain a linear system of $24 n+8$ equations with $24 n+8$ unknowns. The 16 macroscopic decomposed fields can be obtained a posteriori from Eqs. (42)-(45).

\footnotetext{
*romain.corcolle@supelec.fr

†laurent.daniel@supelec.fr

*frederic.bouillault@supelec.fr

${ }^{1}$ IEEE Trans. Ultrason. Ferroelectr. Freq. Control 43 (5), 717 (1996).
}

\footnotetext{
${ }^{2}$ R. L. Goldberg, M. J. Jurgens, D. M. Mills, C. S. Henriquez, D. Vaughan, and S. W. Smith, IEEE Trans. Ultrason. Ferroelectr. Freq. Control 44, 1204 (1997).

${ }^{3}$ M. Besbes, Z. Ren, and A. Razek, IEEE Trans. Magn. 32, 1058 (1996).
} 
${ }^{4}$ J. Lee, J. G. Boyd IV, and D. C. Lagoudas, Int. J. Eng. Sci. 43, 790 (2005).

${ }^{5}$ N. Galopin, X. Mininger, F. Bouillault, and L. Daniel, IEEE Trans. Magn. 44, 834 (2008).

${ }^{6}$ J. E. Huber, N. A. Fleck, C. M. Landis, and R. M. McMeeking, J. Mech. Phys. Solids 47, 1663 (1999).

${ }^{7}$ C.-W. Nan and G. J. Weng, Phys. Rev. B 61, 258 (2000).

${ }^{8}$ A. Haug, J. E. Huber, P. R. Onck, and E. Van der Giessen, J. Mech. Phys. Solids 55, 648 (2007).

${ }^{9}$ C.-W. Nan and G. J. Weng, Phys. Rev. B 60, 6723 (1999).

${ }^{10}$ R. Corcolle, L. Daniel, and F. Bouillault, IEEE Trans. Magn. 44, 17 (2008).

${ }^{11}$ C.-W. Nan, Phys. Rev. B 50, 6082 (1994).

${ }^{12}$ J. Y. Li and M. L. Dunn, Mech. Mater. 31, 149 (1999).

${ }^{13}$ J. H. Huang, Y.-H. Chiu, and H.-K. Liu, J. Appl. Phys. 83, 5364 (1998).
${ }^{14}$ R. Hill, J. Mech. Phys. Solids 13, 89 (1965).

${ }^{15}$ J. W. Hutchinson, Proc. R. Soc. London, Ser. A 319, 247 (1970).

${ }^{16} \mathrm{G}$. W. Milton, The Theory of Composites (Cambridge University Press, New York, 2001).

${ }^{17}$ M. Bornert, T. Bretheau, and P. Gilormini, Homogenization in Mechanics of Materials (Iste, London, 2008).

${ }^{18}$ L. Daniel and R. Corcolle, IEEE Trans. Magn. 43, 3153 (2007).

${ }^{19}$ M. Avellaneda and G. Harshe, J. Intell. Mater. Syst. Struct. 5, 501 (1994).

${ }^{20}$ C.-W. Nan, M. Li, X. Feng, and S. Yu, Appl. Phys. Lett. 78, 2527 (2001).

${ }^{21}$ T. Mura, Micromechanics of defects in solids (Martinus Nijhoff, Boston, 1982).

${ }^{22}$ D. François, A. Pineau, and A. Zaoui, Mechanical Behavior of Materials: Elasticity and Plasticity (Kluwer, Norwell, 1998).

${ }^{23}$ The corresponding MATLAB routines can be obtained on request. 